\title{
Material derivatives of boundary integral operators in electromagnetism and application to inverse scattering problems
}

\author{
Olha Ivanyshyn Yaman ${ }^{1}$ and Frédérique Le Louër ${ }^{2,3}$ \\ ${ }^{1}$ Department of Mathematics, Izmir Institute of Technology, Urla, Izmir, 35430, \\ Turkey \\ ${ }^{2}$ Sorbonne Université, Université de technologie de Compiègne, LMAC EA2222 \\ Laboratoire de Mathématiques Appliquées de Compiègne 60203 Compiègne cedex, \\ France \\ E-mail: olhaivanyshyn@iyte.edu.tr and frederique.le-louer@utc.fr
}

Received 29 December 2015, revised 16 May 2016

Accepted for publication 26 May 2016

Published 5 July 2016

\begin{abstract}
This paper deals with the material derivative analysis of the boundary integral operators arising from the scattering theory of time-harmonic electromagnetic waves and its application to inverse problems. We present new results using the Piola transform of the boundary parametrisation to transport the integral operators on a fixed reference boundary. The transported integral operators are infinitely differentiable with respect to the parametrisations and simplified expressions of the material derivatives are obtained. Using these results, we extend a nonlinear integral equations approach developed for solving acoustic inverse obstacle scattering problems to electromagnetism. The inverse problem is formulated as a pair of nonlinear and ill-posed integral equations for the unknown boundary representing the boundary condition and the measurements, for which the iteratively regularized GaussNewton method can be applied. The algorithm has the interesting feature that it avoids the numerous numerical solution of boundary value problems at each iteration step. Numerical experiments are presented in the special case of star-shaped obstacles.
\end{abstract}

Keywords: electromagnetism, boundary integral equations, material derivatives, inverse obstacle scattering problem

(Some figures may appear in colour only in the online journal)

\footnotetext{
${ }^{3}$ Author to whom any correspondence is addressed. 


\section{Introduction}

The mathematical modeling of physical applications such as non destructive testing, corrosion detection, lens-antennas design or radar and bio-medical imaging leads to inverse boundary value problems. This paper is devoted to extension of a novel inverse scattering method initially developed for solving inverse acoustic scattering problems to electromagnetism. As an example we consider the shape reconstruction problem of a three-dimensional bounded perfectly conducting obstacle from noisy far-field measurements. The whole approach uses geometric optimisation tools and can be adapted to many of the other above-mentioned inverse problems with different kinds of boundary conditions.

We assume the perfect conductor (PC) can be represented by a bounded domain $\Omega$ in $\mathbb{R}^{3}$ with a smooth closed and orientable boundary $\Gamma$. Let $\Omega^{c}$ denote the exterior domain $\mathbb{R}^{3} \backslash \bar{\Omega}$ and $\boldsymbol{n}$ denote the outward unit normal vector to the boundary $\Gamma$. The wavenumber $\kappa$ is a positive real-valued constant. The propagation of electromagnetic waves are governed by the system of Maxwell equations and the time-harmonic Maxwell system can be reduced to a second order equation for the electric field only. In this case the forward problem is formulated as follows: given an incident electric wave $\boldsymbol{E}^{\text {inc }}$ which is assumed to solve the second order Maxwell equation in the absence of any scatterer, find the electric scattered wave $\boldsymbol{E}^{\mathrm{s}}$, solution to the time-harmonic Maxwell equation

$$
\text { curl curl } \boldsymbol{E}^{\mathrm{s}}-\kappa^{2} \boldsymbol{E}^{\mathrm{s}}=0 \quad \text { in } \Omega^{c}
$$

and satisfying the boundary condition,

$$
\boldsymbol{n} \times\left(\boldsymbol{E}^{\mathrm{s}}+\boldsymbol{E}^{\text {inc }}\right)=0 \quad \text { on } \Gamma .
$$

In addition, the scattered field $\boldsymbol{E}^{\mathrm{s}}$, has to satisfy the Silver-Müller radiation condition:

$$
\lim _{|\mathbf{x}| \rightarrow+\infty}|\mathbf{x}|\left|\operatorname{curl} \boldsymbol{E}^{\mathrm{s}}(\mathbf{x}) \times \frac{\mathbf{x}}{|\mathbf{x}|}-i \kappa \boldsymbol{E}^{\mathrm{s}}(\mathbf{x})\right|=0 .
$$

This condition ensures uniqueness of the solution to the scattering problem and guarantees that the scattered wave is outgoing. The PC problem can be reduced in several different ways to a single uniquely solvable Brakhage-Werner type boundary integral equation for all positive values of the exterior wavenumber $\kappa$. The well-posedness of the integral equation and the uniqueness of the solution require the regularization of the single-layer boundary integral operator. Various regularizing operators have been proposed in the scientific literature depending on the applications: they are usually defined as integral operators over $\Gamma[8,9,44]$ or surface differential operators over $\Gamma[13,35]$.

The radiation condition implies that the scattered field $\boldsymbol{E}^{\mathrm{s}}$ has an asymptotic behavior of the form

$$
\boldsymbol{E}^{s}(\mathbf{x})=\frac{e^{i \kappa|\mathbf{x}|}}{|\mathbf{x}|} \boldsymbol{E}^{\infty}(\widehat{\mathbf{x}})+O\left(\frac{1}{|\mathbf{x}|}\right), \quad|\mathbf{x}| \rightarrow \infty
$$

uniformly in all directions $\widehat{\mathbf{x}}=\frac{\mathbf{x}}{|\mathbf{x}|}$. The far-field pattern $\boldsymbol{E}^{\infty}$ is a tangential vector function defined on the unit sphere $\mathbb{S}^{2}$ of $\mathbb{R}^{3}$ and is always analytic. The far-field pattern contains the dependency informations of the solution on the geometry of the obstacle $\Omega$.

Let us consider the scattering of $m$ incident plane waves of the form $\boldsymbol{E}_{k}^{\text {inc }}(\mathbf{x})=\boldsymbol{p}_{k} e^{i \kappa \mathbf{x} \cdot \boldsymbol{d}_{k}}$ where $\boldsymbol{d}_{k}, \boldsymbol{p}_{k} \in \mathbb{S}^{2}$ and $\boldsymbol{d}_{k} \cdot \boldsymbol{p}_{k}=0$. We denote by $F_{k}$ the boundary to far-field operator that 
maps the boundary $\Gamma$ onto the far-field pattern $\boldsymbol{E}_{k}^{\infty}$ of the solution to the forward problem (1.1a)-(1.1c) for the incident wave $\boldsymbol{E}_{k}^{\text {inc }}$.

The inverse PC problem is formulated as follows: given noisy far field measurements $\boldsymbol{E}_{1, \delta}^{\infty}, \ldots, \boldsymbol{E}_{m, \delta}^{\infty}$ obtained from the scattering of the $m$ incident plane waves characterized by the couples of directions and polarizations $\left(\boldsymbol{d}_{k}, \boldsymbol{p}_{k}\right)_{k=1, \ldots, m}$, solve

$$
F_{k}(\Gamma)=\boldsymbol{E}_{k, \delta}^{\infty}, \quad \text { for } k=1, \ldots, m
$$

Here, the noise level is measured in the $\boldsymbol{L}^{2}$-norm, i.e. $\left(\sum_{k=1}^{m}\left\|\boldsymbol{E}_{k, \delta}^{\infty}-\boldsymbol{E}_{k}^{\infty}\right\|_{\boldsymbol{L}^{2}}^{2}\right)^{\frac{1}{2}}<\delta$, and the error bound $\delta$ is assumed to be known. Recently it was proved that a perfect conducting obstacle, which consists of finitely many solid polyhedra, is uniquely determined by the farfield pattern corresponding to a single incident electromagnetic plane wave, see [36]. It is also known that a ball is uniquely determined by the far field pattern for one incident plane wave, see [31]. However, for perfect conductors of general shapes uniqueness is shown only for the case when the far field pattern is known either for all incident directions and polarizations for a fixed wave number, or for all wave numbers contained in some interval for one fixed incident direction and polarization, see [8].

The inverse problem (1.2) is both nonlinear and severely ill-posed. In the scientific literature, one can distinguish two different approaches relying on geometric optimization tools to solve such an inverse problem. The first one consists in reformulating (1.2) as a nonlinear equation posed on an open set of parametrized boundaries $\Gamma_{\boldsymbol{q}}=\boldsymbol{q}(\Gamma)$ with the same genus. Then, we apply a regularized Newton-type method which consists in solving, at each iteration step, the following linearized equation

$$
\partial_{\boldsymbol{q}} \mathcal{F}_{k}[\boldsymbol{q}] \boldsymbol{\xi}=\boldsymbol{E}_{k, \delta}^{\infty}-\mathcal{F}_{k}(\boldsymbol{q})
$$

where $\mathcal{F}_{k}: \boldsymbol{q} \mapsto F_{k}\left(\Gamma_{\boldsymbol{q}}\right)$. The computation of the iterates $\boldsymbol{\xi}$ requires the analysis and an explicit form of the first Fréchet derivative of the parametrized form $\mathcal{F}_{k}$ of the boundary to far-field operator $F_{k}$. The first Fréchet derivative is usually characterized as the far-field pattern of the solution to a new exterior boundary value problem $[11,19,30]$. As a consequence, the inverse scattering algorithm requires multiple numerical solution of boundary integral equations at each iteration step to compute the new iterates by solving a nonlinear least square problem via conjugate gradient method. This method was developed at first for solving inverse obstacle scattering problems in acoustics, see e.g. [20, 22, 24, 29], and it has been recently applied to electromagnetism and elastodynamics by Hohage and Le Louër [23, 34].

The second approach consists in reformulating (1.2) as a pair of nonlinear and ill-posed integral equations for the unknown boundary representing the boundary condition on $\Gamma$ and the far-field pattern on $\mathbb{S}^{2}$. Then, we follow the previous procedure to recover simultaneously the unknown parametrization of the boundary and the density (solution to the boundary integral equation). Pioneering work in this area was conducted by Kress and Rundell in [32]. This novel method requires the differentiability analysis of the classical boundary integral operators with respect to the parametrizations. First investigations were conducted by Potthast [42] in the framework of acoustic scattering and Hölder continuously differentiable function spaces. Later on the results were then generalized to Sobolev spaces by Costabel and Le Louër [10]. To summarize the whole analysis, the integral equations are transported on a reference boundary using a change of variable so that the domain and the range of the integral operators do not depend on the parametrizations anymore and one can use standard differential calculus tools. The Fréchet derivatives of the transported integral operators are called material derivatives and are obtained by differentiating the kernels. The Fréchet derivatives of any order still have the same mapping properties so that one can use the same numerical 
scheme for implementation of these new integral operators. Using these results, Ivanyshyn and Johansson applied this novel method for solving acoustic inverse obstacle scattering problems [25-28]. The algorithm has the significant advantage of avoiding the numerous numerical solution of boundary integral equations at each iteration step, that are replaced by matrix-vector products.

The extension of this novel method for solving electromagnetic inverse obstacle scattering problems is a challenging task. The feasibility of the whole procedure relies on the knowledge of the explicit expressions of the material derivatives, that are suitable for implementation. The differentiability analysis of the standard electromagnetic boundary integral operators poses nontrivial theoretical issues since they are defined for tangential vector densities lying in a Sobolev space of mixed regularity involving the surface divergence operator. Potthast used in [43] projection operators on tangent planes to transport the operators on a fixed reference boundary, but the proposed approach was adapted to the magnetic field integral formulation only. Based on these results, numerical experiments can be found in [8, section 7.3] illustrating the use of the nonlinear integral equations method in inverse electromagnetic scattering. However, considering the integral operators being defined on the above mentioned energy space of mix regularity, the domain and the range of the transported operators still depend on the parametrizations. Costabel and Le Louër [11] get around this difficulty by exploiting the Helmholtz decomposition of the energy space [12]. In counterpart, one has to compute the material derivatives of a family of surface differential operators. In this paper we present an alternative technique which greatly simplifies the previous investigations using the Piola transform of the boundary parametrizations. Furthermore, introducing a new family of regularizing operators in the integral formulations independent of the parametrized boundaries, one gets an additional advantage of avoiding their Frchet differentiation.

The Piola transform is known as a volume transformation which preserves the divergence free condition for a family of parametrized subdomains in $\mathbb{R}^{3}$. This transform was already used in the context of the shape sensitivity analysis of the solution to Navier-Stokes problems $[14,41]$. Here, we extend the mapping properties of the Piola transform to compact closed boundaries and exploit the results to preserve the mixed regularity of the solution to boundary integral equations.

The paper is organized as follows: in section 2, we describe the inverse scattering algorithm based on nonlinear integral equations. We start by deriving the boundary integral equation system equivalent to the inverse problem (1.2). Then, before considering the first order linearization, we show how to transport the whole system to a fixed reference boundary using the Piola transform. The iterative scheme is based on the iteratively regularized GaussNewton (IRGN) method [3, 21, 22]. Section 3 contains the main results of this paper. We give explicit expressions of the material derivatives and discuss their differentiability properties. This work uses some elementary differential geometry tools in order to review the main properties of the Piola transform in a very comprehensive way. The implementation of the iterative algorithm is detailed in section 4 and numerical experiments are presented in section 5 in the special case of star-shaped obstacles. Finally, we draw concluding remarks and we discuss possible research lines in section 6 .

\section{The nonlinear integral equations method}

We denote by $H^{s}(\Gamma)$ the standard complex valued Hilbertian Sobolev space of order $s \in \mathbb{R}$ defined on $\Gamma$ with the convention $H^{0}=L^{2}$. Spaces of vector functions are denoted by 
boldface letters, thus $\boldsymbol{H}^{s}=\left(H^{s}\right)^{3}$ and, spaces of tangent vectors fields are characterized by the lower index t. For $k \in \mathbb{N}$, we denote by $\mathscr{C}^{k}\left(\bar{\Omega}, \mathbb{R}^{3}\right)$ the space of $k$-times continuously differentiable functions from $\bar{\Omega}$ to $\mathbb{R}^{3}$. We denote by $\mathscr{C}^{k, \alpha}\left(\bar{\Omega}, \mathbb{R}^{3}\right)$ the class of functions in $\mathscr{C}^{k}\left(\bar{\Omega}, \mathbb{R}^{3}\right)$ whose all the partial derivatives of order $k$ are $\alpha$-Hölder continuous with $0<\alpha \leqslant 1$. Finally, we define the spaces $\mathscr{C}^{k}\left(\Gamma, \mathbb{R}^{3}\right)$ and $\mathscr{C}^{k, \alpha}\left(\Gamma, \mathbb{R}^{3}\right)$ as the set of the restrictions to $\Gamma$ of any function in $\mathscr{C}^{k}\left(\bar{\Omega}, \mathbb{R}^{3}\right)$ and $\mathscr{C}^{k, \alpha}\left(\bar{\Omega}, \mathbb{R}^{3}\right)$, respectively.

We use the following surface differential operators: the tangential gradient is denoted by $\operatorname{grad}_{\Gamma}$, the surface divergence is denoted by $\operatorname{div}_{\Gamma}$, the tangential vector curl is denoted by curl $_{\Gamma}$ and the surface scalar curl is denoted by curl ${ }_{\Gamma}$. For their definitions we refer to [37, pages 68-75] or [23, appendix A]. For any vector field $\boldsymbol{q} \in \mathscr{C}^{1}\left(\Gamma, \mathbb{R}^{3}\right)$, we denote by $\left[\mathrm{D}_{\Gamma} \boldsymbol{q}\right]$ the 3 by 3 matrix function whose $j$ th line is the tangential gradient of the $j$ th component of $\boldsymbol{q}$. We introduce the Hilbert space

$$
\boldsymbol{H}_{\mathrm{div}}^{-\frac{1}{2}}(\Gamma)=\left\{\boldsymbol{j} \in \boldsymbol{H}^{-\frac{1}{2}}(\Gamma) ; \boldsymbol{j} \cdot \boldsymbol{n}=0 \text { and } \operatorname{div}_{\Gamma} \boldsymbol{j} \in H^{-\frac{1}{2}}(\Gamma)\right\}
$$

endowed with the norm $\|\cdot\|_{\boldsymbol{H}_{\text {div }}^{-\frac{1}{2}}(\Gamma)}=\left(\|\cdot\|_{\boldsymbol{H}^{-\frac{1}{2}(\Gamma)}}^{2}+\left\|\operatorname{div}_{\Gamma} \cdot\right\|_{H^{-\frac{1}{2}}(\Gamma)}^{2}\right)^{1 / 2}$. The dual space of $\boldsymbol{H}_{\mathrm{div}}^{-\frac{1}{2}}(\Gamma)$ for the $\boldsymbol{L}_{\mathrm{t}}^{2}$ duality product is

$$
\boldsymbol{H}_{\text {curl }}^{-\frac{1}{2}}(\Gamma)=\left\{\boldsymbol{j} \in \boldsymbol{H}^{-\frac{1}{2}}(\Gamma) ; \boldsymbol{j} \cdot \boldsymbol{n}=0 \text { and } \operatorname{curl}_{\Gamma} \boldsymbol{j} \in H^{-\frac{1}{2}}(\Gamma)\right\}
$$

and the exterior product with the normal vector defines a bicontinuous isomorphism between $\boldsymbol{H}_{\mathrm{div}}^{-\frac{1}{2}}(\Gamma)$ and $\boldsymbol{H}_{\text {curl }}^{-\frac{1}{2}}(\Gamma)$.

Let $\kappa$ be a positive real-valued wavenumber and $\Phi(\kappa, \mathbf{z})=\frac{e^{i \kappa|\mathbf{z}|}}{4 \pi|\mathbf{z}|}$ be the fundamental solution of the Helmholtz equation $\Delta u+\kappa^{2} u=0$. For any solution $\boldsymbol{E}^{s}$ to the Maxwell equation $(1.1 a)$ that satisfies the radiation condition $(1.1 c)$, it holds the Stratton-Chu representation formula for $\mathbf{x} \in \Omega^{c}[8$, chapter 6$]$ :

$$
\begin{aligned}
\boldsymbol{E}^{s}(\mathbf{x})= & \int_{\Gamma} \operatorname{curl}^{\mathbf{x}}\left\{\Phi(\kappa, \mathbf{x}-\mathbf{y})\left(\boldsymbol{n}(\mathbf{y}) \times \boldsymbol{E}^{s}(\mathbf{y})\right)\right\} d s(\mathbf{y}) \\
& +\frac{1}{\kappa^{2}} \int_{\Gamma} \operatorname{curl}^{\operatorname{curl}}{ }^{\mathbf{x}}\left\{\Phi(\kappa, \mathbf{x}-\mathbf{y})\left(\boldsymbol{n}(\mathbf{y}) \times \operatorname{curl} \boldsymbol{E}^{s}(\mathbf{y})\right)\right\} d s(\mathbf{y})
\end{aligned}
$$

Conversely, for any $\boldsymbol{j} \in \boldsymbol{H}_{\mathrm{div}}^{-\frac{1}{2}}(\Gamma)$ the potentials

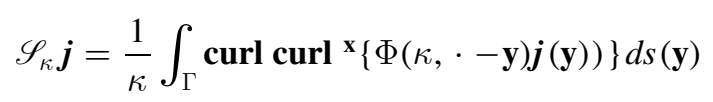

and

$$
\mathscr{D}_{\kappa} \boldsymbol{j}=\int_{\Gamma} \operatorname{curl}^{\mathbf{x}}\{\Phi(\kappa, \cdot-\mathbf{y}) \boldsymbol{j}(\mathbf{y})\} d s(\mathbf{y})
$$

satisfy the Maxwell equation and the Silver-Müller radiation condition. Using these results, the forward problem $(1.1 a)-(1.1 c)$ can be reduced, in several different ways, to a single uniquely solvable boundary integral equation. We will consider the following indirect approach. It is based on the layer ansatz:

$$
\boldsymbol{E}^{s}=\mathscr{D}_{\kappa} \boldsymbol{j}+i \eta \mathscr{S}_{\kappa} \boldsymbol{\Lambda} \boldsymbol{j}
$$


where $\boldsymbol{\Lambda}$ is a bounded operator from $\boldsymbol{H}_{\mathrm{div}}^{-\frac{1}{2}}(\Gamma)$ to itself, self-adjoint and elliptic for the bilinear form

$$
(\boldsymbol{j}, \boldsymbol{m}) \mapsto \int_{\Gamma} \boldsymbol{j} \cdot(\boldsymbol{n} \times \boldsymbol{m}) d s
$$

and $\eta$ is a non vanishing real constant. By the jump relations, the field $\boldsymbol{E}^{\mathrm{s}}$ given by (2.1) solves the Dirichlet boundary value problem $(1.1 a)-(1.1 c)$ if the density $\boldsymbol{j}$ solves the following integral equation

$$
\left(\mathrm{I}+M_{\kappa}+i \eta C_{\kappa} \boldsymbol{\Lambda}\right) \boldsymbol{j}=-2\left(\boldsymbol{n} \times \boldsymbol{E}_{\mid \Gamma}^{i n c}\right) \quad \text { on } \Gamma .
$$

Here the single layer potential $C_{\kappa}$ and the double layer potential $M_{\kappa}$ are defined by

$$
\begin{aligned}
& M_{\kappa} \boldsymbol{j}(\mathbf{x})=\int_{\Gamma} \boldsymbol{n}(\mathbf{x}) \times \operatorname{curl}^{\mathbf{x}}\{2 \Phi(\kappa, \mathbf{x}-\mathbf{y}) \boldsymbol{j}(\mathbf{y})\} d s(\mathbf{y}), \\
& C_{\kappa} \boldsymbol{j}(\mathbf{x})=\frac{1}{\kappa} \int_{\Gamma} \boldsymbol{n}(\mathbf{x}) \times \operatorname{curl} \operatorname{curl}^{\mathbf{x}}\{2 \Phi(\kappa, \mathbf{x}-\mathbf{y}) \boldsymbol{j}(\mathbf{y})\} d s(\mathbf{y}) \\
& =\kappa \boldsymbol{n}(\mathbf{x}) \times \int_{\Gamma} 2 \Phi(\kappa, \mathbf{x}-\mathbf{y}) \boldsymbol{j}(\mathbf{y}) d s(\mathbf{y})-\frac{1}{\kappa} \operatorname{curl}{ }_{\Gamma} \int_{\Gamma} 2 \Phi(\kappa, \mathbf{x}-\mathbf{y}) \operatorname{div}_{\Gamma} \boldsymbol{j}(\mathbf{y}) d s(\mathbf{y}) .
\end{aligned}
$$

The operator $M_{\kappa}: \boldsymbol{H}_{\mathrm{div}}^{-\frac{1}{2}}(\Gamma) \rightarrow \boldsymbol{H}_{\mathrm{div}}^{-\frac{1}{2}}(\Gamma)$ is compact (for smooth boundaries) and the operator $C_{\kappa}$ has a hypersingular kernel but it is bounded on $\boldsymbol{H}_{\mathrm{div}}^{-\frac{1}{2}}(\Gamma)$. The operator $\boldsymbol{\Lambda}$ is then chosen such that $\left(\mathrm{I}+M_{\kappa}+i \eta C_{\kappa} \boldsymbol{\Lambda}\right)$ is a Fredholm operator of index zero. One can find in the scientific literature various definitions for the operator $\boldsymbol{\Lambda}$. Kress first proposed in [8, theorem 6.21] a compact regularization $\boldsymbol{\Lambda} \boldsymbol{j}=\boldsymbol{n} \times S_{0}^{2} \boldsymbol{j}$ where $S_{0}$ is the single layer boundary integral operator associated with the Laplace equation, thus (I $\left.+M_{\kappa}+i \eta C_{\kappa} \Lambda\right)$ is a Fredholm operator of the second kind. One can also use the elliptic and invertible operator which is a variant of the operator $C_{\kappa}$ [44] defined on $\boldsymbol{H}_{\mathrm{div}}^{-\frac{1}{2}}(\Gamma)$ by $\boldsymbol{\Lambda} \boldsymbol{j}=\boldsymbol{n} \times S_{0} \boldsymbol{j}+\operatorname{curl}{ }_{\Gamma} S_{0} \operatorname{div}_{\Gamma} \boldsymbol{j}$. We obtain a well-posed integral equation for Lipschitz domains. Another choice is to use an approximation of the magnetic-to-electric field operator which is suitable for solving highfrequency scattering [13]. We point out that the operator $\boldsymbol{\Lambda}$ usually depends on the boundary $\Gamma$ and can be chosen to obtain a well-posed integral equation on $\boldsymbol{H}_{\mathrm{div}}^{-\frac{1}{2}}(\Gamma) \cap \boldsymbol{L}_{\mathrm{t}}^{2}(\Gamma)$.

The far-field pattern can be computed via the integral representation formula

$$
\boldsymbol{E}^{\infty}=\mathscr{D}_{\kappa}^{\infty} \boldsymbol{j}+i \eta \mathscr{S}_{\kappa}^{\infty} \boldsymbol{\Lambda} \boldsymbol{j}
$$

where the far-field operator $\mathscr{S}_{\kappa}^{\infty}$ and $\mathscr{D}_{\kappa}^{\infty}$ are defined for $\boldsymbol{j} \in \boldsymbol{H}_{\mathrm{div}}^{-\frac{1}{2}}(\Gamma)$ and $\widehat{\mathbf{x}} \in \mathbb{S}^{2}$ by:

$$
\begin{aligned}
& \mathscr{S}_{\kappa}^{\infty} \boldsymbol{j}(\widehat{\mathbf{x}})=\frac{\kappa}{4 \pi} \int_{\Gamma} e^{-i \kappa \hat{\mathbf{x}} \cdot \mathbf{y}}((\widehat{\mathbf{x}} \times \boldsymbol{j}(\mathbf{y})) \times \widehat{\mathbf{x}}) d s(\mathbf{y}), \\
& \mathscr{D}_{\kappa}^{\infty} \boldsymbol{j}(\widehat{\mathbf{x}})=\frac{i \kappa}{4 \pi} \int_{\Gamma} e^{-i \kappa \hat{\mathbf{x}} \cdot \mathbf{y}}(\widehat{\mathbf{x}} \times \boldsymbol{j}(\mathbf{y})) d s(\mathbf{y}) .
\end{aligned}
$$

Setting $\mathrm{I}_{o p}=\left(\mathrm{I}+M_{\kappa}+i \eta C_{\kappa} \Lambda\right)$ and $\mathscr{F}^{\infty}=\mathscr{D}_{\kappa}^{\infty}+i \eta \mathscr{S}_{\kappa}^{\infty} \boldsymbol{\Lambda}$, the boundary to far-field operator can be factorized as $F_{k}(\Gamma)=\mathscr{F}^{\infty} \mathrm{I}_{o p}^{-1}\left(-2\left(\boldsymbol{n} \times \boldsymbol{E}_{k, \mid \Gamma}^{i n c}\right)\right)$ and the inverse problem (1.2) is equivalent to the following systems of nonlinear and still ill-posed integral equations for the unknown boundary $\Gamma$

$$
\left\{\begin{array}{ll}
\mathrm{I}_{o p} \boldsymbol{j}_{k}=-2\left(\boldsymbol{n} \times \boldsymbol{E}_{k \mid \Gamma}^{i n c}\right), & \text { on } \Gamma \\
\mathscr{F}^{\infty} \boldsymbol{j}_{k}=\boldsymbol{E}_{k, \delta}^{\infty}, & \text { on } \mathbb{S}^{2}
\end{array} \quad \text { for } k=1, \ldots, m .\right.
$$

Instead of recovering only $\Gamma$, we seak the solutions $\boldsymbol{j}_{1}, \ldots, \boldsymbol{j}_{m}$ to the boundary integral equations and the boundary $\Gamma$ simultaneously. 
To remove the ill-posedness of the system (2.5), we choose a fixed reference domain $\Omega_{\text {ref }}$ with a closed and orientable boundary $\Gamma_{\text {ref }}$ of class $\mathscr{C}^{1, \alpha}$ at least, with $0<\alpha \leqslant 1$. Moreover, we consider variations generated by transformations of the form $\mathbf{x} \mapsto \boldsymbol{q}(\mathbf{x})$ with a point $\mathbf{x}$ in the space $\mathbb{R}^{3}$ and a smooth vector function $\boldsymbol{q}$ defined in a neighborhood of $\Gamma_{\text {ref }}$. The function $(\boldsymbol{q}-\mathrm{I})$ is assumed to be sufficiently small elements of the Banach space $\mathscr{C}^{1, \alpha}\left(\Gamma, \mathbb{R}^{3}\right)$. In this way, $\boldsymbol{q}$ is a diffeomorphism from $\Gamma_{\text {ref }}$ to $\Gamma_{\boldsymbol{q}}=\left\{\boldsymbol{q}(\mathbf{x}) ; \mathbf{x} \in \Gamma_{\text {ref }}\right\}$, so that the surface $\Gamma_{\boldsymbol{q}}$ is still a smooth boundary of a domain $\Omega_{\boldsymbol{q}}$ with same genus as $\Omega_{\text {ref. }}$. We have the continuous embedding $\boldsymbol{H}^{s}(\Gamma) \hookrightarrow \mathscr{C}^{1, \alpha}\left(\Gamma, \mathbb{R}^{3}\right)$ for any $s>2+\alpha$ (see [1, p 98] and [37, p 50]). We choose $s>2$ (i.e. $0<\alpha<s-2$ ) and we define the following open set of admissible variations

$$
\mathcal{Q}_{a d} \subset\left\{\boldsymbol{q} \in \boldsymbol{H}^{s}\left(\Gamma_{\mathrm{ref}}\right) ; \Gamma_{\boldsymbol{q}} \text { is diffeomorphic to } \Gamma_{\mathrm{ref}}\right\} .
$$

By $\boldsymbol{n}_{\boldsymbol{q}}$ we denote the outward unit normal vector to $\Gamma_{\boldsymbol{q}}$ and, in what follows, we will distinguish the quantities related to the perfect conductor problem for the domain $\Omega_{\boldsymbol{q}}$ through the index $\boldsymbol{q}$. When $\boldsymbol{q}=\mathrm{I}$, we remove the index $\boldsymbol{q}$.

The operators $\mathrm{I}_{o p}$ and $\mathscr{F}^{\infty}$ are now considered as functions acting from $\mathcal{Q}_{a d}$ to $\mathscr{L}\left(\boldsymbol{H}_{\mathrm{div}}^{-\frac{1}{2}}\left(\Gamma_{\boldsymbol{q}}\right), \boldsymbol{H}_{\mathrm{div}}^{-\frac{1}{2}}\left(\Gamma_{\boldsymbol{q}}\right)\right)$ and $\mathscr{L}\left(\boldsymbol{H}_{\mathrm{div}}^{-\frac{1}{2}}\left(\Gamma_{\boldsymbol{q}}\right), \boldsymbol{L}_{\mathrm{t}}^{2}\left(\mathbb{S}^{2}\right)\right)$, respectively, and we rewrite the system (2.5) as follows:

$$
\left\{\begin{array}{ll}
\mathrm{I}_{o p}(\boldsymbol{q}) \boldsymbol{j}_{k, \boldsymbol{q}}=-2\left(\boldsymbol{n}_{\boldsymbol{q}} \times \boldsymbol{E}_{k \mid \Gamma_{q}}^{i n c}\right), & \text { on } \Gamma_{\boldsymbol{q}} \\
\mathscr{F}^{\infty}(\boldsymbol{q}) \boldsymbol{j}_{k, \boldsymbol{q}}=\boldsymbol{E}_{k, \delta}^{\infty}, & \text { on } \mathbb{S}^{2},
\end{array} \quad \text { for } k=1, \ldots, m .\right.
$$

The objective is to solve the nonlinear system (2.6) for unknowns $\boldsymbol{q}$ and $\boldsymbol{j}_{\boldsymbol{q}}$ by applying the IRGN method for Hilbert spaces [22]. It requires the description of the differentiability properties of the boundary integral operators. For this purpose, we have to remove the dependence on $\boldsymbol{q}$ in the domain and in the range of the operators $\mathrm{I}_{o p}(\boldsymbol{q})$ and $\mathscr{F}^{\infty}(\boldsymbol{q})$. Indeed, these operators are both defined on the $\boldsymbol{q}$-dependent space $\boldsymbol{H}_{\mathrm{div}}^{-\frac{1}{2}}\left(\Gamma_{\boldsymbol{q}}\right)$ which poses nontrivial problems for their differentiability analysis with respect to $\boldsymbol{q}$. This issue was already considered by Costabel and Le Louër in [11]. The strategy consists in introducing a transformation $\mathcal{P}_{\boldsymbol{q}}$ that maps the variable space $\boldsymbol{H}_{\mathrm{div}}^{-\frac{1}{2}}\left(\Gamma_{\boldsymbol{q}}\right)$ onto $\boldsymbol{H}_{\mathrm{div}}^{-\frac{1}{2}}\left(\Gamma_{\mathrm{ref}}\right)$ and to rewrite the system (2.6) as follows

$$
\left\{\begin{array}{ll}
\mathcal{P}_{\boldsymbol{q}} \mathrm{I}_{o p}(\boldsymbol{q}) \mathcal{P}_{\boldsymbol{q}}^{-1} \boldsymbol{j}_{k}=\boldsymbol{f}_{k}(\boldsymbol{q}), & \text { on } \Gamma_{\text {ref }} \\
\mathscr{F}^{\infty}(\boldsymbol{q}) \mathcal{P}_{\boldsymbol{q}}^{-1} \boldsymbol{j}_{k}=\boldsymbol{E}_{k, \delta}^{\infty}, & \text { on } \mathbb{S}^{2}
\end{array} \quad \text { for } k=1, \ldots, m,\right.
$$

where $\boldsymbol{j}_{k}=\mathcal{P}_{\boldsymbol{q}} \boldsymbol{j}_{k, \boldsymbol{q}} \in \boldsymbol{H}_{\mathrm{div}}^{-\frac{1}{2}}\left(\Gamma_{\mathrm{ref}}\right)$ and $\boldsymbol{f}_{k}(\boldsymbol{q})=-2 \mathcal{P}_{\boldsymbol{q}}\left(\boldsymbol{n}_{\boldsymbol{q}} \times \boldsymbol{E}_{k \mid \Gamma_{\boldsymbol{q}}}^{\text {inc }}\right) \in \boldsymbol{H}_{\mathrm{div}}^{-\frac{1}{2}}\left(\Gamma_{\mathrm{ref}}\right)$. We are finally led to study the differentiability properties of the following operators:

$$
\begin{aligned}
\widehat{\mathrm{I}}_{o p}: \mathcal{Q}_{a d} & \rightarrow \mathscr{L}\left(\boldsymbol{H}_{\mathrm{div}}^{-\frac{1}{2}}\left(\Gamma_{\mathrm{ref}}\right), \boldsymbol{H}_{\mathrm{div}}^{-\frac{1}{2}}\left(\Gamma_{\mathrm{ref}}\right)\right) \\
\boldsymbol{q} & \mapsto \mathcal{P}_{\boldsymbol{q}} \mathrm{I}_{o p}(\boldsymbol{q}) \mathcal{P}_{\boldsymbol{q}}^{-1},
\end{aligned}
$$

and

$$
\begin{aligned}
\widehat{\mathscr{F}}^{\infty}: \mathcal{Q}_{a d} & \rightarrow \mathscr{L}\left(\boldsymbol{H}_{\mathrm{div}}^{-\frac{1}{2}}\left(\Gamma_{\mathrm{ref}}\right), \boldsymbol{L}_{\mathrm{t}}^{2}\left(\mathbb{S}^{2}\right)\right) \\
\boldsymbol{q} & \mapsto \mathscr{F}^{\infty}(\boldsymbol{q}) \mathcal{P}_{\boldsymbol{q}}^{-1} .
\end{aligned}
$$

To construct the transformation $\mathcal{P}_{\boldsymbol{q}}$, Costabel and Le Louër [10, 11] exploited the Hodge structure [12] of $\boldsymbol{H}_{\mathrm{div}}^{-\frac{1}{2}}\left(\Gamma_{\boldsymbol{q}}\right)=\left(\operatorname{grad}_{\Gamma_{\boldsymbol{q}}} H^{\frac{3}{2}}\left(\Gamma_{\boldsymbol{q}}\right)\right) \oplus\left(\operatorname{curl}_{\Gamma_{\boldsymbol{q}}} H^{\frac{1}{2}}\left(\Gamma_{\boldsymbol{q}}\right)\right)$ for smooth and simply 
connected closed boundaries. On one hand, we are lead to the differentiability analysis of some boundary integral operators lying in the classical Sobolev spaces. On the other hand, we have to study the differentiability properties with respect to $\boldsymbol{q}$ of the following surface differential operators : $\operatorname{grad}_{\Gamma_{q}}, \operatorname{curl} \Gamma_{\Gamma_{q}}, \operatorname{div}_{\Gamma_{q}}, \operatorname{curl} \Gamma_{\Gamma_{q}}, \Delta_{\Gamma_{q}}$ and its inverse. The resulting expression of the first Fréchet derivative of $\hat{\mathrm{I}}_{o p}$ is rather complicated.

For numerical purposes, it is more convenient to use instead the Piola transform of $\boldsymbol{q}$ defined as follows

$$
\begin{aligned}
\mathcal{P}_{\boldsymbol{q}}: \boldsymbol{H}_{\mathrm{div}}^{-\frac{1}{2}}\left(\Gamma_{\boldsymbol{q}}\right) & \rightarrow \boldsymbol{H}_{\mathrm{div}}^{-\frac{1}{2}}\left(\Gamma_{\mathrm{ref}}\right) \\
\boldsymbol{j}_{\boldsymbol{q}} & \mapsto \boldsymbol{j}=J_{\boldsymbol{q}}\left[\mathrm{D}_{\Gamma_{\mathrm{ref}}} \boldsymbol{q}\right]^{-1}\left(\boldsymbol{j}_{\boldsymbol{q}} \circ \boldsymbol{q}\right),
\end{aligned}
$$

where we have set $\left[\mathrm{D}_{\Gamma_{\mathrm{ref}}} \boldsymbol{q}\right]^{-1}=\left[\mathrm{D}_{\Gamma_{q}} \boldsymbol{q}^{-1}\right] \circ \boldsymbol{q}$ and $J_{\boldsymbol{q}}$ is the determinant of the Jacobian matrix of the change of variable $\mathbf{x} \mapsto \boldsymbol{q}(\mathbf{x})$. The main properties of $\mathcal{P}_{\boldsymbol{q}}$ are given in section 3 . To describe the iterative scheme for solving (2.7), we use the following notations $\widehat{\mathrm{I}}_{o p}(\boldsymbol{q}) \boldsymbol{j}=\widehat{\mathrm{I}}_{o p}(\boldsymbol{q}, \boldsymbol{j})$ and $\widehat{\mathscr{F}}^{\infty}(\boldsymbol{q}) \boldsymbol{j}=\widehat{\mathscr{F}}^{\infty}(\boldsymbol{q}, \boldsymbol{j})$. The method involves the full linearization of the system of integral equations (2.7) with respect to both the boundary parametrization and the density. After linearization we obtain for $k=1, \ldots, m$

$$
\begin{array}{ll}
\widehat{\mathrm{I}}_{o p}\left(\boldsymbol{q}, \boldsymbol{j}_{k}\right)+\widehat{\mathrm{I}}_{o p}\left(\boldsymbol{q}, \boldsymbol{m}_{k}\right)+\partial_{\boldsymbol{q}} \widehat{\mathrm{I}}_{o p}\left[\boldsymbol{q}, \boldsymbol{j}_{k}\right] \boldsymbol{\xi}=\boldsymbol{f}_{k}(\boldsymbol{q})+\partial_{\boldsymbol{q}} \boldsymbol{f}_{k}[\boldsymbol{q}] \boldsymbol{\xi}, & \text { on } \Gamma_{\text {ref }} \\
\widehat{\mathscr{F}}^{\infty}\left(\boldsymbol{q}, \boldsymbol{j}_{k}\right)+\widehat{\mathscr{F}}^{\infty}\left(\boldsymbol{q}, \boldsymbol{m}_{k}\right)+\partial_{\boldsymbol{q}} \widehat{\mathscr{F}}^{\infty}\left[\boldsymbol{q}, \boldsymbol{j}_{k}\right] \boldsymbol{\xi}=\boldsymbol{E}_{k, \delta}^{\infty}, & \text { on } \mathbb{S}^{2} .
\end{array}
$$

We rearrange the system (2.9), for $k=1, \ldots, m$, in the form

$$
A_{k}\left(\begin{array}{c}
\boldsymbol{m}_{k} \\
\xi
\end{array}\right)=B_{k}
$$

where

$A_{k}=\left(\begin{array}{cc}\widehat{\mathrm{I}}_{o p}(\boldsymbol{q}, \cdot) & \partial_{\boldsymbol{q}} \widehat{\mathrm{I}}_{o p}\left[\boldsymbol{q}, \boldsymbol{j}_{k}\right]-\partial_{\boldsymbol{q}} \boldsymbol{f}_{k}[\boldsymbol{q}] \\ \widehat{\mathscr{F}}^{\infty}(\boldsymbol{q}, \cdot) & \partial_{\boldsymbol{q}} \widehat{\mathscr{F}}^{\infty}\left[\boldsymbol{q}, \boldsymbol{j}_{k}\right]\end{array}\right), \quad B_{k}=\left(\begin{array}{c}\boldsymbol{f}_{k}(\boldsymbol{q})-\widehat{\mathrm{I}}_{o p}\left(\boldsymbol{q}, \boldsymbol{j}_{k}\right) \\ \boldsymbol{E}_{k, \delta}^{\infty}-\widehat{\mathscr{F}}^{\infty}\left(\boldsymbol{q}, \boldsymbol{j}_{k}\right)\end{array}\right)$.

One can prove that solving (1.3) is equivalent to solving the system of integral equations $(2.10 a)-(2.10 b)$. The proof is detailed in [39] using the magnetic field integral equation. Here we avoid the existence of real-valued eigenfrequencies using BraquageWerner type boundary integral equations.

Theorem 2.1. Let $\boldsymbol{q} \in \mathcal{Q}_{a d}$ and set $\boldsymbol{j}_{k}=\left[\hat{\mathrm{I}}_{o p}(\boldsymbol{q}, \cdot)\right]^{-1} \boldsymbol{f}_{k}[\boldsymbol{q}]$. If $\boldsymbol{\xi}$ solves (1.3), then $\boldsymbol{\xi}$ and $\boldsymbol{m}_{k}=-\left[\hat{\mathrm{I}}_{o p}(\boldsymbol{q})\right]^{-1}\left(\partial_{\boldsymbol{q}} \hat{\mathrm{I}}_{o p}\left[\boldsymbol{q}, \boldsymbol{j}_{k}\right] \boldsymbol{\xi}-\partial_{\boldsymbol{q}} \boldsymbol{f}_{k}[\boldsymbol{q}] \boldsymbol{\xi}\right)$ solve the system (2.10a)-(2.10b). Conversely, if $\boldsymbol{\xi}$ and $\boldsymbol{m}_{k}$ solve (2.10a)-(2.10b), then $\boldsymbol{\xi}$ solves (1.3).

We deduce that the injectivity and the denseness of the operator $A_{k}$ is related to the injectivity and the denseness of the operator $\partial_{\boldsymbol{q}} \mathcal{F}_{k}[\boldsymbol{q}]$. Contrary to the acoustic case [28], it is still an open question whether or not the null-space of the operator $\partial_{q} \mathcal{F}_{k}[q]$ is reduced to the set $\mathcal{N}:=\left\{\boldsymbol{\xi} \in \mathscr{C}^{1, \alpha}\left(\Gamma_{\text {ref }}, \mathbb{R}^{3}\right) ;\left(\boldsymbol{\xi} \circ \boldsymbol{q}^{-1}\right) \cdot \boldsymbol{n}_{\boldsymbol{q}}=0\right\}$ when $\boldsymbol{E}_{k}^{\text {inc }}$ is an incident plane wave. Counterexamples can be found in [19] when $\boldsymbol{E}_{k}^{i n c}$ is a Maxwell eigenfunction. As a consequence we cannot prove yet that the restriction to starlike domains is sufficient to obtain injectivity.

To start the procedure we make an initial guess for the unknown boundary $\Gamma$ parametrized by $\boldsymbol{q}^{0}=\boldsymbol{q}_{\delta}^{0}$. We compute the intitial density $\boldsymbol{j}_{k}^{0}=\boldsymbol{j}_{k, \delta}^{0}$ by solving (2.3) on $\Gamma_{\boldsymbol{q}_{\delta}^{0}}$. Then, at each iteration step, knowing the current approximation $\left(\boldsymbol{j}_{k, \delta}^{N}, \boldsymbol{q}_{\delta}^{N}\right)$ we compute the next 
iterates $\boldsymbol{j}_{1, \delta}^{N+1}, \ldots, \boldsymbol{j}_{m, \delta}^{N+1}$ and $\boldsymbol{q}_{\delta}^{N+1}$ by solving the nonlinear least square problem

$$
\begin{aligned}
\left(\begin{array}{c}
\boldsymbol{j}_{1, \delta}^{N+1} \\
\vdots \\
\boldsymbol{j}_{m, \delta}^{N+1} \\
\boldsymbol{q}_{\delta}^{N+1}
\end{array}\right):=\underset{\left(\boldsymbol{j}_{1}, \ldots, \boldsymbol{j}_{m}, \boldsymbol{q}\right)}{\operatorname{argmin}}\left[\sum_{k=1}^{m}\left\|A_{k, \delta}^{N}\left(\begin{array}{c}
\boldsymbol{j}_{k}-\boldsymbol{j}_{k, \delta}^{N} \\
\boldsymbol{q}-\boldsymbol{q}_{\delta}^{N}
\end{array}\right)-B_{k, \delta}^{N}\right\|^{2}\right. \\
\left.+\alpha_{N}\left\|\left(\boldsymbol{q}-\boldsymbol{q}^{0}\right)\right\|^{2}+\beta_{N} \sum_{k=1}^{m}\left\|\left(\boldsymbol{j}_{k}-\boldsymbol{j}_{k}^{0}\right)\right\|^{2}\right],
\end{aligned}
$$

via the conjugate gradient method. The solutions of boundary value problems when using the classical Newton iteration (1.3) are replaced by matrix-vector products. Here the regularization parameters are chosen of the form $\alpha_{N}=\gamma^{-N} \alpha_{0}$ and $\beta_{N}=\gamma^{-N} \beta_{0}$, with $\gamma>1$, which provides logarithmic convergence rates of the IRGN method in Hilbert spaces $[21,22$, theorem 4.9] when the stopping rule is given by the Morozov's discrepancy principle

$$
\left(\sum_{k=1}^{m}\left\|\mathcal{F}_{k}\left(\boldsymbol{q}_{\delta}^{N}\right)-\boldsymbol{E}_{k}^{\infty}\right\|_{\boldsymbol{L}^{2}}^{2}\right)^{\frac{1}{2}} \leqslant \tau \delta
$$

where $\tau>1$ is known from the beginning. The corrections $\boldsymbol{m}_{k, \delta}^{N}=\boldsymbol{j}_{k, \delta}^{N+1}-\boldsymbol{j}_{k, \delta}^{N}$ and $\boldsymbol{\xi}_{\delta}^{N}=\boldsymbol{q}_{\delta}^{N+1}-\boldsymbol{q}_{\delta}^{N}$ are the unique solution to the regularized linear equation [3]

$$
\left[\left(\begin{array}{cc}
\beta_{N} \mathrm{I}_{\left(\boldsymbol{L}_{\mathrm{t}}^{2}\right)^{m}} & 0 \\
0 & \alpha_{N} \mathrm{I}_{\boldsymbol{H}^{s}}
\end{array}\right)+\left[\mathbf{A}_{\delta}^{N}\right]^{*} \mathbf{A}_{\delta}^{N}\right]\left(\begin{array}{c}
\boldsymbol{m}_{1, \delta}^{N} \\
\vdots \\
\boldsymbol{m}_{m, \delta}^{N} \\
\boldsymbol{\xi}_{\delta}^{N}
\end{array}\right)=\left[\mathbf{A}_{\delta}^{N}\right]^{*} \mathbf{B}_{\delta}^{N}+\left(\begin{array}{c}
\beta_{N}\left(\boldsymbol{j}_{1, \delta}^{N}-\boldsymbol{j}_{1}^{0}\right) \\
\vdots \\
\beta_{N}\left(\boldsymbol{j}_{m, \delta}^{N}-\boldsymbol{j}_{m}^{0}\right) \\
\alpha_{N}\left(\boldsymbol{q}_{\delta}^{N}-\boldsymbol{q}_{\delta}^{0}\right)
\end{array}\right)
$$

where $\left[\mathbf{A}_{\delta}^{N}\right]^{*}$ is the $\boldsymbol{L}^{2}$ adjoint operator of $\mathbf{A}_{\delta}^{N}, \mathbf{B}_{\delta}^{N}={ }^{\top}\left(B_{1, \delta}^{N}, \ldots, B_{m, \delta}^{N}\right)$ and

$$
\mathbf{A}_{\delta}^{N}=\left(\begin{array}{cccccc}
\widehat{\mathrm{I}}_{o p}\left(\boldsymbol{q}_{\delta}^{N}, \cdot\right) & 0 & \ldots & \ldots & 0 & \partial_{\boldsymbol{q}} \widehat{\mathrm{I}}_{o p}\left[\boldsymbol{q}_{\delta}^{N}, \boldsymbol{j}_{1, \delta}^{N}\right]-\partial_{\boldsymbol{q}} \boldsymbol{f}_{1}\left[\boldsymbol{q}_{\delta}^{N}\right] \\
\widehat{\mathscr{F}}^{\infty}\left(\boldsymbol{q}_{\delta}^{N}, \cdot\right) & 0 & \ddots & & \vdots & \partial_{\boldsymbol{q}} \widehat{\mathscr{F}}^{\infty}\left[\boldsymbol{q}_{\delta}^{N}, \boldsymbol{j}_{1, \delta}^{N}\right] \\
0 & & & \ddots & \vdots & \vdots \\
\vdots & \ddots & & & 0 & \vdots \\
\vdots & & \ddots & & \widehat{\mathrm{I}}_{o p}\left(\boldsymbol{q}_{\delta}^{N}, \cdot\right) & \partial_{\boldsymbol{q}} \widehat{\mathrm{I}}_{o p}\left[\boldsymbol{q}_{\delta}^{N}, \boldsymbol{j}_{m, \delta}^{N}\right]-\partial_{\boldsymbol{q}} \boldsymbol{f}_{m}\left[\boldsymbol{q}_{\delta}^{N}\right] \\
0 & \ldots & \ldots & 0 & \widehat{\mathscr{F}}^{\infty}\left(\boldsymbol{q}_{\delta}^{N}, \cdot\right) & \partial_{\boldsymbol{q}} \widehat{\mathscr{F}}^{\infty}\left[\boldsymbol{q}_{\delta}^{N}, \boldsymbol{j}_{m, \delta}^{N}\right]
\end{array}\right) .
$$

\section{Material derivative analysis of the integral operators}

The Piola transform has been already considered by Hohage and Le Louër in [23], when $\Gamma_{\text {ref }}=\mathbb{S}^{2}$ for solving the dielectric scattering problem. In particular, using a projection method on the orthonormal system of tangential vector spherical harmonics, the systems of second-kind boundary integral equations were implemented. Therefore, this section uses notations and contains some results stated in [23, section 3] which are extended to a general orientable surface $\Gamma_{\text {ref }}$ below. In addition, the mapping properties of the adjoint operator of the Piola transform are stated. 
We recall elementary results on differential geometry following [37, pp 68-71]. Given $\mathbf{x} \in \Gamma_{\text {ref }}$, we assume that the tangent plane $\boldsymbol{T}_{\mathbf{x}}$ to $\Gamma_{\text {ref }}$ at $\mathbf{x}$ is generated by the tangential contravariant vectors $\boldsymbol{e}_{1}(\mathbf{x})$ and $\boldsymbol{e}_{2}(\mathbf{x})$ and the cotangent plane $\boldsymbol{T}_{\mathbf{x}}^{*}$ to $\Gamma_{\text {ref }}$ at $\mathbf{x}$ is generated by the tangential covariant vectors $\boldsymbol{e}^{1}(\mathbf{x})$ and $\boldsymbol{e}^{2}(\mathbf{x})$ such that $\left\|\boldsymbol{e}_{1} \times \boldsymbol{e}_{2}\right\|=1$ and $\boldsymbol{e}_{i}(\mathbf{x}) \cdot \boldsymbol{e}^{j}(\mathbf{x})=\delta_{i}^{j}$ for $i, j=1,2$. Here $\delta_{i}^{j}$ represents the Kronecker symbol. In this case, we have $\boldsymbol{n}=\boldsymbol{e}_{1} \times \boldsymbol{e}_{2}$.

Let $\boldsymbol{q} \in \mathcal{Q}_{a d}$. The surface Jacobian matrix $\left[\mathrm{D}_{\Gamma_{\mathrm{ref}}} \boldsymbol{q}(\mathbf{x})\right]$ maps the tangent plane $\boldsymbol{T}_{\mathbf{x}}$ to $\Gamma_{\text {ref }}$ at the point $\mathbf{x}$ onto the tangent plane $\boldsymbol{T}_{\boldsymbol{q}(\mathbf{x})}$ to $\Gamma_{\boldsymbol{q}}$ at the point $\boldsymbol{q}(\mathbf{x})$. The latter is generated by the contravariant vectors

$$
\boldsymbol{t}_{1}(\boldsymbol{q} ; \mathbf{x})=\left[\mathrm{D}_{\Gamma_{\mathrm{ref}}} \boldsymbol{q}(\mathbf{x})\right] \boldsymbol{e}_{1}(\mathbf{x}) \text { and } \boldsymbol{t}_{2}(\boldsymbol{q} ; \mathbf{x})=\left[\mathrm{D}_{\Gamma_{\mathrm{ref}}} \boldsymbol{q}(\mathbf{x})\right] \boldsymbol{e}_{2}(\mathbf{x}) .
$$

Thus, we can write $\left[\mathrm{D}_{\Gamma_{\mathrm{ref}}} \boldsymbol{q}\right]=\boldsymbol{t}_{1}(\boldsymbol{q}) \otimes \boldsymbol{e}^{1}+\boldsymbol{t}_{2}(\boldsymbol{q}) \otimes \boldsymbol{e}^{2}$. The functions $J_{\boldsymbol{q}}$ and $\boldsymbol{n}_{\boldsymbol{q}} \circ \boldsymbol{q}$ can be computed via the formulas $J_{\boldsymbol{q}}=\left|\boldsymbol{t}_{1} \times \boldsymbol{t}_{2}\right|$ and $\boldsymbol{n}_{\boldsymbol{q}} \circ \boldsymbol{q}=\frac{\boldsymbol{t}_{1} \times \boldsymbol{t}_{2}}{J_{q}}$. The parametrization $\boldsymbol{q}: \Gamma_{\mathrm{ref}} \rightarrow \Gamma$ being a diffeomorphism, we set $\left[\mathrm{D}_{\Gamma_{\text {ref }}} \boldsymbol{q}(\mathbf{x})\right]^{-1}=\left[\mathrm{D}_{\Gamma_{q}} \boldsymbol{q}^{-1}\right] \circ \boldsymbol{q}(\mathbf{x})$. The transposed matrix ${ }^{\mathrm{T}}\left[\mathrm{D}_{\Gamma_{\mathrm{ref}}} \boldsymbol{q}(\mathbf{x})\right]^{-1}$ maps the cotangent plane $\boldsymbol{T}_{\mathbf{x}}^{*}$ to $\Gamma_{\text {ref }}$ at the point $\mathbf{x}$ onto the cotangent plane $\boldsymbol{T}_{\boldsymbol{q}(\mathbf{x})}^{*}$ to $\Gamma_{\boldsymbol{q}}$ at the point $\boldsymbol{q}(\mathbf{x})$. The latter is generated by the covariant vectors

$$
\begin{aligned}
& \boldsymbol{t}^{1}(\boldsymbol{q} ; \mathbf{x})={ }^{\mathrm{T}}\left[\mathrm{D}_{\Gamma_{\mathrm{ref}}} \boldsymbol{q}(\mathbf{x})\right]^{-1} \boldsymbol{e}^{1}(\mathbf{x})=\frac{\boldsymbol{t}_{2}(\boldsymbol{q} ; \mathbf{x}) \times \boldsymbol{n}_{\boldsymbol{q}}(\boldsymbol{q}(\mathbf{x}))}{J_{\boldsymbol{q}}(\mathbf{x})} \\
& \boldsymbol{t}^{2}(\boldsymbol{q} ; \mathbf{x})={ }^{\mathrm{T}}\left[\mathrm{D}_{\Gamma_{\mathrm{ref}}} \boldsymbol{q}(\mathbf{x})\right]^{-1} \boldsymbol{e}^{2}(\mathbf{x})=\frac{\left(\boldsymbol{n}_{\boldsymbol{q}}(\boldsymbol{q}(\mathbf{x})) \times \boldsymbol{t}_{1}(\boldsymbol{q} ; \mathbf{x})\right.}{J_{\boldsymbol{q}}(\mathbf{x})}
\end{aligned}
$$

Hence, we can write ${ }^{\mathrm{T}}\left[\mathrm{D}_{\Gamma_{\text {ref }}} \boldsymbol{q}(\mathbf{x})\right]^{-1}=\boldsymbol{t}^{1}(\boldsymbol{q}) \otimes \boldsymbol{e}_{1}+\boldsymbol{t}^{2}(\boldsymbol{q}) \otimes \boldsymbol{e}_{2}$. The adjoint operator of $\mathcal{P}_{\boldsymbol{q}}$ for the $\boldsymbol{L}_{\mathrm{t}}^{2}$ duality product is defined by

$$
\begin{aligned}
\mathcal{P}_{\boldsymbol{q}}^{*}: \boldsymbol{H}_{\text {curl }}^{-\frac{1}{2}}\left(\Gamma_{\mathrm{ref}}\right) & \rightarrow \boldsymbol{H}_{\text {curl }}^{-\frac{1}{2}}\left(\Gamma_{\boldsymbol{q}}\right) \\
\boldsymbol{j} & \mapsto \boldsymbol{j}_{\boldsymbol{q}}=\left({ }^{\top}\left[\mathrm{D}_{\Gamma_{\mathrm{ref}}} \boldsymbol{q}\right]^{-1} \boldsymbol{j}\right) \circ \boldsymbol{q}^{-1} .
\end{aligned}
$$

For any tangential vector functions $j$ to $\Gamma_{\text {ref }}$ and $j_{q}$ to $\Gamma_{q}$ we have the following useful identities

$$
\mathcal{P}_{\boldsymbol{q}}^{*}(\boldsymbol{n} \times \boldsymbol{j})=\boldsymbol{n}_{\boldsymbol{q}} \times\left(\mathcal{P}_{\boldsymbol{q}}^{-1} \boldsymbol{j}\right) \quad \text { and } \quad \boldsymbol{n} \times\left(\mathcal{P}_{\boldsymbol{q}} \boldsymbol{j}_{\boldsymbol{q}}\right)=\mathcal{P}_{\boldsymbol{q}}^{-*}\left(\boldsymbol{n}_{\boldsymbol{q}} \times \boldsymbol{j}_{\boldsymbol{q}}\right) .
$$

From this and the definition of the surface differential operators using an atlas given in [37, pp 72-75] or [23, appendix A], it is straightforward to deduce the following transformation formulas for the surface differential operators. Let $u \in \mathscr{C}^{1}\left(\Gamma_{\boldsymbol{q}}, \mathbb{R}\right)$ and $\boldsymbol{v} \in \mathscr{C}^{1}\left(\Gamma_{\boldsymbol{q}}, \mathbb{R}^{3}\right)$, then we have [23, section 3]:

$$
\begin{aligned}
& \mathcal{P}_{\boldsymbol{q}}^{-*}\left(\operatorname{grad}_{\Gamma_{\boldsymbol{q}}} u\right)=\operatorname{grad}_{\Gamma_{\mathrm{ref}}}(u \circ \boldsymbol{q}), \quad \mathcal{P}_{\boldsymbol{q}}\left(\operatorname{curl}_{\Gamma_{\boldsymbol{q}}} u\right)=\operatorname{curl}_{\Gamma_{\mathrm{ref}}}(u \circ \boldsymbol{q}), \\
& J_{\boldsymbol{q}}\left(\operatorname{div}_{\Gamma_{\boldsymbol{q}}} \boldsymbol{v}\right) \circ \boldsymbol{q}=\operatorname{div}_{\Gamma_{\mathrm{ref}}}\left(\mathcal{P}_{\boldsymbol{q}} \boldsymbol{v}\right), \quad J_{\boldsymbol{q}}\left(\operatorname{curl}_{\Gamma_{\boldsymbol{q}}} \boldsymbol{v}\right) \circ \boldsymbol{q}=\operatorname{curl}_{\Gamma_{\mathrm{ref}}}\left(\mathcal{P}_{\boldsymbol{q}}^{-*} \boldsymbol{v}\right)
\end{aligned}
$$

Furthermore, we have [4, proposition 4.7]

$$
\begin{aligned}
& \operatorname{curl}_{\Gamma_{\mathrm{ref}}} u=\left(\operatorname{grad}_{\Gamma_{\mathrm{ref}}} u\right) \times \boldsymbol{n}, \operatorname{curl} \Gamma_{\mathrm{ref}} \boldsymbol{v}=\operatorname{div}_{\Gamma_{\mathrm{ref}}}(\boldsymbol{v} \times \boldsymbol{n}), \\
& \operatorname{curl}_{\Gamma_{\mathrm{ref}}} \operatorname{grad}_{\Gamma_{\mathrm{ref}}} u=0, \quad \operatorname{div}_{\Gamma_{\mathrm{ref}}} \operatorname{curl}{ }_{\Gamma_{\mathrm{ref}}} u=0 .
\end{aligned}
$$

By density arguments, these formulas can be extended to Sobolev spaces.

From the formulas (3.3) and (3.4) we deduce the following lemma, which is of great importance for the shape sensitivity analysis in electromagnetism. Parts of the proof are stated 
in [23] using the Hodge decomposition of the $\boldsymbol{H}_{\operatorname{div}_{\Gamma}}^{-\frac{1}{2}}\left(\mathbb{S}^{2}\right)$ and can be extended to more general surfaces using the Hodge decompositions [4, equations (29) and (33)] and [5, theorem 3.4].

Lemma 3.1. Let $\boldsymbol{q} \in \mathcal{Q}_{\text {ad. }}$. The Piola transform $\mathcal{P}_{\boldsymbol{q}}$ of $\boldsymbol{q}$, and its adjoint operator $\mathcal{P}_{\boldsymbol{q}}^{*}$, defined by (2.8) and (3.1) respectively, are bicontinuous invertible operators. Moreover, we have

$$
\begin{aligned}
\mathcal{P}_{\boldsymbol{q}}\left(\operatorname{Ker}\left[\operatorname{div}_{\Gamma_{\boldsymbol{q}}}\left(\boldsymbol{H}_{\mathrm{t}}^{-\frac{1}{2}}\left(\Gamma_{\boldsymbol{q}}\right)\right]\right)\right. & =\operatorname{Ker}\left[\operatorname{div}_{\Gamma_{\mathrm{ref}}}\left(\boldsymbol{H}_{\mathrm{t}}^{-\frac{1}{2}}\left(\Gamma_{\mathrm{ref}}\right)\right]\right. \\
\operatorname{Ker}\left[\operatorname{curl}_{\Gamma_{\boldsymbol{q}}}\left(\boldsymbol{H}_{\mathrm{t}}^{-\frac{1}{2}}\left(\Gamma_{\boldsymbol{q}}\right)\right]\right. & =\mathcal{P}_{\boldsymbol{q}}^{*}\left(\operatorname{Ker}\left[\operatorname{curl}_{\Gamma_{\mathrm{ref}}}\left(\boldsymbol{H}_{\mathrm{t}}^{-\frac{1}{2}}\left(\Gamma_{\mathrm{ref}}\right)\right]\right)\right.
\end{aligned}
$$

The remainder of the section is devoted to the material derivative analysis of the operators $\widehat{\mathrm{I}}_{o p}$ and $\widehat{\mathscr{F}}^{\infty}$ that can be written as follows

$$
\begin{aligned}
\hat{\mathrm{I}}_{o p}(\boldsymbol{q}) & =\mathrm{I}_{\boldsymbol{H}_{\mathrm{div}}^{-\frac{1}{2}}\left(\Gamma_{\mathrm{ref}}\right)}+\left(\mathcal{P}_{\boldsymbol{q}} M_{\kappa}(\boldsymbol{q}) \mathcal{P}_{\boldsymbol{q}}^{-1}\right)+i \eta\left(\mathcal{P}_{\boldsymbol{q}} C_{\kappa}(\boldsymbol{q}) \mathcal{P}_{\boldsymbol{q}}^{-1}\right)\left(\mathcal{P}_{\boldsymbol{q}} \boldsymbol{\Lambda}(\boldsymbol{q}) \mathcal{P}_{\boldsymbol{q}}^{-1}\right), \\
\widehat{\mathscr{F}}^{\infty}(\boldsymbol{q}) & =\mathscr{D}_{\kappa}^{\infty} \mathcal{P}_{\boldsymbol{q}}^{-1}+i \eta\left(\mathscr{S}_{\kappa}^{\infty} \mathcal{P}_{\boldsymbol{q}}^{-1}\right)\left(\mathcal{P}_{\boldsymbol{q}} \boldsymbol{\Lambda}(\boldsymbol{q}) \mathcal{P}_{\boldsymbol{q}}^{-1}\right)
\end{aligned}
$$

Let $\boldsymbol{j}_{\boldsymbol{q}} \in \boldsymbol{H}_{\mathrm{div}}^{-\frac{1}{2}}\left(\Gamma_{\boldsymbol{q}}\right), \boldsymbol{j}=\mathcal{P}_{\boldsymbol{q}} \boldsymbol{j}_{\boldsymbol{q}}$ and $\widehat{\Lambda}(\boldsymbol{q})=\left(\mathcal{P}_{\boldsymbol{q}} \boldsymbol{\Lambda}(\boldsymbol{q}) \mathcal{P}_{\boldsymbol{q}}^{-1}\right)$. Then we have

$$
\begin{aligned}
\int_{\Gamma_{q}} \boldsymbol{\Lambda}(\boldsymbol{q}) \boldsymbol{j}_{\boldsymbol{q}} \cdot\left(\boldsymbol{n}_{\boldsymbol{q}} \times \boldsymbol{j}_{\boldsymbol{q}}\right) d s & =\int_{\Gamma_{\boldsymbol{q}}}\left(\mathcal{P}_{\boldsymbol{q}}^{-1} \widehat{\boldsymbol{\Lambda}}(\boldsymbol{q}) \mathcal{P}_{\boldsymbol{q}} \boldsymbol{j}_{\boldsymbol{q}}\right) \cdot\left(\boldsymbol{n}_{\boldsymbol{q}} \times \boldsymbol{j}_{\boldsymbol{q}}\right) d s \\
& =\int_{\Gamma_{\mathrm{ref}}}\left(\widehat{\boldsymbol{\Lambda}}(\boldsymbol{q}) \mathcal{P}_{\boldsymbol{q}} \boldsymbol{j}_{\boldsymbol{q}}\right) \cdot \mathcal{P}_{\boldsymbol{q}}^{-*}\left(\boldsymbol{n}_{\boldsymbol{q}} \times \boldsymbol{j}_{\boldsymbol{q}}\right) d s \\
& =\int_{\Gamma_{\mathrm{ref}}}(\widehat{\boldsymbol{\Lambda}}(\boldsymbol{q}) \boldsymbol{j}) \cdot(\boldsymbol{n} \times \boldsymbol{j}) d s
\end{aligned}
$$

We deduce that the operator $\boldsymbol{\Lambda}(\boldsymbol{q})$ is an elliptic operator on $\boldsymbol{H}_{\mathrm{div}}^{-\frac{1}{2}}\left(\Gamma_{\boldsymbol{q}}\right)$ for the bilinear form (2.2) with $\Gamma=\Gamma_{\boldsymbol{q}}$ if and only if $\widehat{\boldsymbol{\Lambda}}(\boldsymbol{q})$ is an elliptic operator on $\boldsymbol{H}_{\mathrm{div}}^{-\frac{1}{2}}\left(\Gamma_{\mathrm{ref}}\right)$ for the bilinear form (2.2) with $\Gamma=\Gamma_{\text {ref }}$. Moreover, the operators $\Lambda(q)$ and $\widehat{\Lambda}(q)$ have the same mapping properties. It follows that we can choose the regularizing operator $\Lambda(q)$ such that $\widehat{\Lambda}(q)$ does not depend on $\boldsymbol{q}$ and we write $\widehat{\Lambda}(q)=\widehat{\Lambda}$.

Proposition 3.2. The mappings $\boldsymbol{t}_{k}: \boldsymbol{q} \in \mathcal{Q}_{a d} \rightarrow \boldsymbol{t}_{k}(\boldsymbol{q}) \in \mathscr{C}^{0, \alpha}\left(\Gamma_{\mathrm{ref}}, \mathbb{R}^{3}\right)$, for $k=1,2$ are Fréchet differentiable at all $\boldsymbol{q} \in \mathcal{Q}_{a d}$ and the first derivatives at $\boldsymbol{q}$ in the direction $\boldsymbol{\xi} \in \mathscr{C}^{1, \alpha}\left(\Gamma_{\text {ref }}\right)$ are given by

$$
\left.\left(\partial_{\boldsymbol{q}} \boldsymbol{t}_{k}[\boldsymbol{q}] \boldsymbol{\xi}\right)(\mathbf{x})\right)=\left[\mathrm{D}_{\Gamma_{\mathrm{ref}}} \boldsymbol{\xi}(\mathbf{x})\right] \boldsymbol{e}_{k}(\mathbf{x}), \quad \text { for } k=1,2 .
$$

Proof. Let $\boldsymbol{q} \in \mathcal{Q}_{a d}$ and $\boldsymbol{\xi} \in \mathscr{C}^{1, \alpha}\left(\Gamma_{\text {ref }}, \mathbb{R}^{3}\right)$ such that $\boldsymbol{q}+\boldsymbol{\xi} \in \mathcal{Q}_{a d}$. By definition of the tangent vectors $\boldsymbol{t}_{k}$ for $k=1,2$ we have:

$$
\boldsymbol{t}_{k}(\boldsymbol{q}+\boldsymbol{\xi})-\boldsymbol{t}_{k}(\boldsymbol{q})=\left[\mathrm{D}_{\Gamma_{\mathrm{ref}}}(\boldsymbol{q}+\boldsymbol{\xi})\right] \boldsymbol{e}_{k}-\left[\mathrm{D}_{\Gamma_{\mathrm{ref}}} \boldsymbol{q}\right] \boldsymbol{e}_{k}=\left[\mathrm{D}_{\Gamma_{\mathrm{ref}}} \boldsymbol{\xi}\right] \boldsymbol{e}_{k} .
$$

The function $\boldsymbol{t}_{k}$ is linear and continuous at $\boldsymbol{q}$, therefore its first Fréchet derivative at $\boldsymbol{q}$ is $\boldsymbol{t}_{k}$ and the higher order Fréchet derivatives vanish. 
Let $\boldsymbol{q} \in \mathcal{Q}_{a d}$ and $\boldsymbol{j} \in \boldsymbol{H}_{\mathrm{div}}^{-\frac{1}{2}}\left(\Gamma_{\mathrm{ref}}\right)$. We set $\widehat{M}_{\kappa}(\boldsymbol{q}, \boldsymbol{j})=\mathcal{P}_{\boldsymbol{q}} \boldsymbol{M}_{\kappa}(\boldsymbol{q}) \mathcal{P}_{\boldsymbol{q}}^{-1} \boldsymbol{j}$ and we have

$$
\begin{aligned}
\widehat{M}_{\kappa}(\boldsymbol{q}, \boldsymbol{j})(\mathbf{x})= & J_{q}(\mathbf{x})\left[\mathrm{D}_{\Gamma_{\mathrm{ref}}} \boldsymbol{q}(\mathbf{x})\right]^{-1} \int_{\Gamma_{\mathrm{ref}}} \boldsymbol{n}_{\boldsymbol{q}}(\boldsymbol{q}(\mathbf{x})) \\
& \times \operatorname{curl}^{\boldsymbol{q}(\mathbf{x})}\left\{2 \Phi(\kappa, \boldsymbol{q}(\mathbf{x})-\boldsymbol{q}(\mathbf{y}))\left[\mathrm{D}_{\Gamma_{\mathrm{ref}}} \boldsymbol{q}(\mathbf{y})\right] \boldsymbol{j}(\mathbf{y})\right\} d s(\mathbf{y}) .
\end{aligned}
$$

From (3.2) and (3.3), this can be rewritten

$$
\begin{aligned}
\widehat{M}_{\kappa}(\boldsymbol{q}, \boldsymbol{j})(\mathbf{x})= & \boldsymbol{n}(\mathbf{x}) \times \int_{\Gamma_{\mathrm{ref}}}{ }^{\mathrm{T}}\left[\mathrm{D}_{\Gamma_{\mathrm{ref}}} \boldsymbol{q}(\mathbf{x})\right] \operatorname{curl} \boldsymbol{q}(\mathbf{x})\left\{2 \Phi(\kappa, \boldsymbol{q}(\mathbf{x})-\boldsymbol{q}(\mathbf{y}))\left[\mathrm{D}_{\Gamma_{\mathrm{ref}}} \boldsymbol{q}(\mathbf{y})\right] \boldsymbol{j}(\mathbf{y})\right\} d s(\mathbf{y}) \\
= & \boldsymbol{e}^{1}(\mathbf{x}) \int_{\Gamma_{\mathrm{ref}}}\left\{\mathcal{M}_{1}^{1}(\boldsymbol{q} ; \mathbf{x}, \mathbf{y})\left(\boldsymbol{j}(\mathbf{y}) \cdot \boldsymbol{e}_{1}(\mathbf{y})\right)+\mathcal{M}_{2}^{1}(\boldsymbol{q} ; \mathbf{x}, \mathbf{y})\left(\boldsymbol{j}(\mathbf{y}) \cdot \boldsymbol{e}_{2}(\mathbf{y})\right)\right\} d s(\mathbf{y}) \\
& +\boldsymbol{e}^{2}(\mathbf{x}) \int_{\Gamma_{\mathrm{ref}}}\left\{\mathcal{M}_{1}^{2}(\boldsymbol{q} ; \mathbf{x}, \mathbf{y})\left(\boldsymbol{j}(\mathbf{y}) \cdot \boldsymbol{e}_{1}(\mathbf{y})\right)\right. \\
& \left.+\mathcal{M}_{2}^{2}(\boldsymbol{q} ; \mathbf{x}, \mathbf{y})\left(\boldsymbol{j}(\mathbf{y}) \cdot \boldsymbol{e}_{2}(\mathbf{y})\right)\right\} d s(\mathbf{y})
\end{aligned}
$$

where

$$
\begin{aligned}
& \mathcal{M}_{1}^{1}(\boldsymbol{q} ; \mathbf{x}, \mathbf{y})=-2\left(\operatorname{grad}^{\mathbf{z}} \Phi\right)(\kappa, \boldsymbol{q}(\mathbf{x})-\boldsymbol{q}(\mathbf{y})) \cdot\left(\boldsymbol{t}_{1}(\boldsymbol{q} ; \mathbf{y}) \times \boldsymbol{t}_{2}(\boldsymbol{q} ; \mathbf{x})\right) \\
& \mathcal{M}_{2}^{1}(\boldsymbol{q} ; \mathbf{x}, \mathbf{y})=-2\left(\operatorname{grad}^{\mathbf{z}} \Phi\right)(\kappa, \boldsymbol{q}(\mathbf{x})-\boldsymbol{q}(\mathbf{y})) \cdot\left(\boldsymbol{t}_{2}(\boldsymbol{q} ; \mathbf{y}) \times \boldsymbol{t}_{2}(\boldsymbol{q} ; \mathbf{x})\right) \\
& \mathcal{M}_{1}^{2}(\boldsymbol{q} ; \mathbf{x}, \mathbf{y})=2\left(\operatorname{grad}^{\mathbf{z}} \Phi\right)(\kappa, \boldsymbol{q}(\mathbf{x})-\boldsymbol{q}(\mathbf{y})) \cdot\left(\boldsymbol{t}_{1}(\boldsymbol{q} ; \mathbf{y}) \times \boldsymbol{t}_{1}(\boldsymbol{q} ; \mathbf{x})\right) \\
& \mathcal{M}_{2}^{2}(\boldsymbol{q} ; \mathbf{x}, \mathbf{y})=2\left(\operatorname{grad}^{\mathbf{z}} \Phi\right)(\kappa, \boldsymbol{q}(\mathbf{x})-\boldsymbol{q}(\mathbf{y})) \cdot\left(\boldsymbol{t}_{2}(\boldsymbol{q} ; \mathbf{y}) \times \boldsymbol{t}_{1}(\boldsymbol{q} ; \mathbf{x})\right)
\end{aligned}
$$

with

$$
\operatorname{grad}^{\mathbf{z}} \Phi(\kappa, \mathbf{z})=\frac{\Phi(\kappa, \mathbf{z})}{|\mathbf{z}|}\left(i \kappa-\frac{1}{|\mathbf{z}|}\right) \mathbf{z}
$$

Remark 3.3. Boundary integral operators resulting from classical scattering theory are divided into three classes of operators depending of the behaviour on their pseudohomogeneous kernel when $\|\mathbf{x}-\mathbf{y}\| \rightarrow 0$ that is $k(\mathbf{x}, \mathbf{y})=O\left(\|\mathbf{x}-\mathbf{y}\|^{\beta-2}\right)$ [37, section 4.3]. For smooth boundaries in $\mathbb{R}^{3}$, (i) if $\beta>0$ then the kernel is said to be weakly singular and the operator is bounded and compact from $H^{-\frac{1}{2}}(\Gamma)$ to $H^{-\frac{1}{2}}(\Gamma)$. For example, the kernels $\mathcal{M}_{i}^{j}$, for $i, j=1,2$, are weakly singular with $\beta=\alpha$. Indeed, for any $(\mathbf{x}, \mathbf{y}) \in \Gamma_{\text {ref }} \times \Gamma_{\text {ref }}$, the $\alpha$-Hölder continuity of $\boldsymbol{q}$ allows us to write

$$
\begin{aligned}
\boldsymbol{q}(\mathbf{y})-\boldsymbol{q}(\mathbf{x}) & =\left[\mathrm{D}_{\Gamma_{\mathrm{ref}}} \boldsymbol{q}(\mathbf{x})\right](\mathbf{y}-\mathbf{x})+O\left(\|\mathbf{x}-\mathbf{y}\|^{1+\alpha}\right) \text { and } \\
\boldsymbol{t}_{k}(\boldsymbol{q} ; \mathbf{y}) & =\boldsymbol{t}_{k}(\boldsymbol{q} ; \mathbf{x})+O\left(\|\mathbf{x}-\mathbf{y}\|^{\alpha}\right)
\end{aligned}
$$

We use $\quad \boldsymbol{t}_{1}(\boldsymbol{q} ; \mathbf{x}) \times \boldsymbol{t}_{2}(\boldsymbol{q} ; \mathbf{x})=J_{\boldsymbol{q}}(\mathbf{x}) \boldsymbol{n}_{\boldsymbol{q}}(\boldsymbol{q}(\mathbf{x})), \quad \boldsymbol{n}_{\boldsymbol{q}}(\boldsymbol{q}(\mathbf{x})) \cdot\left[\mathrm{D}_{\Gamma_{\mathrm{ref}}} \boldsymbol{q}(\mathbf{x})\right](\mathbf{y}-\mathbf{x})=0 \quad$ and $\boldsymbol{t}_{k}(\boldsymbol{q} ; \mathbf{x}) \times \boldsymbol{t}_{k}(\boldsymbol{q} ; \mathbf{x})=0$;

(ii) if $\beta=0$ and the kernel is odd, then the kernel is said to be strongly singular and the operator is bounded from $H^{-\frac{1}{2}}(\Gamma)$ to itself;

(iii) if $\beta<0$ then the kernel is said to be hypersingular and the operator is unbounded on $H^{-\frac{1}{2}}(\Gamma)$. In this case we usually rewrite the kernel involving surface differential operators. 
Theorem 3.4. The mapping $\widehat{M}_{k}: \mathcal{Q}_{a d} \rightarrow \mathscr{L}\left(\boldsymbol{H}_{\mathrm{div}}^{-\frac{1}{2}}\left(\Gamma_{\mathrm{ref}}\right)\right)$ is Fréchet differentiable at all $\boldsymbol{q} \in \mathcal{Q}_{\text {ad }}$ and the first derivatives at $\boldsymbol{q}$ in the direction $\boldsymbol{\xi} \in \mathscr{C}^{1, \alpha}\left(\Gamma_{\text {ref }}\right)$ is a bounded linear integral operator in $\mathscr{L}\left(\boldsymbol{H}_{\mathrm{div}}^{-\frac{1}{2}}\left(\Gamma_{\mathrm{ref}}\right)\right)$ defined for $\boldsymbol{j} \in \boldsymbol{H}_{\mathrm{div}}^{-\frac{1}{2}}\left(\Gamma_{\mathrm{ref}}\right)$ by

$$
\begin{aligned}
\left(\partial_{\boldsymbol{q}} \widehat{M}_{\kappa}[\boldsymbol{q}, \boldsymbol{j}] \boldsymbol{\xi}\right)(\mathbf{x})= & \boldsymbol{e}^{1}(\mathbf{x}) \int_{\Gamma_{\mathrm{ref}}}\left\{\left(\partial_{\boldsymbol{q}} \mathcal{M}_{1}^{1}[\boldsymbol{q}] \boldsymbol{\xi}\right)(\mathbf{x}, \mathbf{y})\left(\boldsymbol{j}(\mathbf{y}) \cdot \boldsymbol{e}_{1}(\mathbf{y})\right)\right. \\
& \left.+\left(\partial_{\boldsymbol{q}} \mathcal{M}_{2}^{1}[\boldsymbol{q}] \boldsymbol{\xi}\right)(\mathbf{x}, \mathbf{y})\left(\boldsymbol{j}(\mathbf{y}) \cdot \boldsymbol{e}_{2}(\mathbf{y})\right)\right\} d s(\mathbf{y}) \\
& +\boldsymbol{e}^{2}(\mathbf{x}) \int_{\Gamma_{\mathrm{ref}}}\left\{\left(\partial_{\boldsymbol{q}} \mathcal{M}_{1}^{2}[\boldsymbol{q}] \boldsymbol{\xi}\right)(\mathbf{x}, \mathbf{y})\left(\boldsymbol{j}(\mathbf{y}) \cdot \boldsymbol{e}_{1}(\mathbf{y})\right)\right. \\
& \left.+\left(\partial_{\boldsymbol{q}} \mathcal{M}_{2}^{2}[\boldsymbol{q}] \boldsymbol{\xi}\right)(\mathbf{x}, \mathbf{y})\left(\boldsymbol{j}(\mathbf{y}) \cdot \boldsymbol{e}_{2}(\mathbf{y})\right)\right\} d s(\mathbf{y})
\end{aligned}
$$

where

$$
\begin{aligned}
\left(\partial_{\boldsymbol{q}} \mathcal{M}_{1}^{1}[\boldsymbol{q}] \boldsymbol{\xi}\right)(\mathbf{x}, \mathbf{y})= & -2\left\{\left[\left(\mathbf{H e s s}^{\mathbf{z}} \Phi\right)(\kappa, \boldsymbol{q}(\mathbf{x})-\boldsymbol{q}(\mathbf{y}))\right](\boldsymbol{\xi}(\mathbf{x})-\boldsymbol{\xi}(\mathbf{y}))\right\} \\
& \cdot\left(\boldsymbol{t}_{1}(\boldsymbol{q} ; \mathbf{y}) \times \boldsymbol{t}_{2}(\boldsymbol{q} ; \mathbf{x})\right)-2\left(\operatorname{grad}^{\mathbf{z}} \Phi\right)(\kappa, \boldsymbol{q}(\mathbf{x})-\boldsymbol{q}(\mathbf{y})) \\
& \cdot\left(\left(\partial_{\boldsymbol{q}} \boldsymbol{t}_{1}[\boldsymbol{q}] \boldsymbol{\xi}\right)(\mathbf{y}) \times \boldsymbol{t}_{2}(\boldsymbol{q} ; \mathbf{x})+\boldsymbol{t}_{1}(\boldsymbol{q} ; \mathbf{y})\right. \\
& \left.\times\left(\partial_{\boldsymbol{q}} \boldsymbol{t}_{2}[\boldsymbol{q}] \boldsymbol{\xi}\right)(\mathbf{x})\right), \\
\left(\partial_{\boldsymbol{q}} \mathcal{M}_{2}^{1}[\boldsymbol{q}] \boldsymbol{\xi}\right)(\mathbf{x}, \mathbf{y})= & -2\left\{\left[\left(\mathbf{H e s s}^{\mathbf{z}} \Phi\right)(\kappa, \boldsymbol{q}(\mathbf{x})-\boldsymbol{q}(\mathbf{y}))\right](\boldsymbol{\xi}(\mathbf{x})-\boldsymbol{\xi}(\mathbf{y}))\right\} \\
& \cdot\left(\boldsymbol{t}_{2}(\boldsymbol{q} ; \mathbf{y}) \times \boldsymbol{t}_{2}(\boldsymbol{q} ; \mathbf{x})\right)-2\left(\mathbf{g r a d}^{\mathbf{z}} \Phi\right)(\kappa, \boldsymbol{q}(\mathbf{x})-\boldsymbol{q}(\mathbf{y})) \\
& \cdot\left(\left(\partial_{\boldsymbol{q}} \boldsymbol{t}_{2}[\boldsymbol{q}] \boldsymbol{\xi}\right)(\mathbf{y}) \times \boldsymbol{t}_{2}(\boldsymbol{q} ; \mathbf{x})+\boldsymbol{t}_{2}(\boldsymbol{q} ; \mathbf{y})\right. \\
& \left.\times\left(\partial_{\boldsymbol{q}} \boldsymbol{t}_{2}[\boldsymbol{q}] \boldsymbol{\xi}\right)(\mathbf{x})\right), \\
\left(\partial_{\boldsymbol{q}} \mathcal{M}_{1}^{2}[\boldsymbol{q}] \boldsymbol{\xi}\right)(\mathbf{x}, \mathbf{y})= & \left.2\left\{\left(\mathbf{H e s s}^{\mathbf{z}} \Phi\right)(\kappa, \boldsymbol{q}(\mathbf{x})-\boldsymbol{q}(\mathbf{y}))\right](\boldsymbol{\xi}(\mathbf{x})-\boldsymbol{\xi}(\mathbf{y}))\right\} \\
& \cdot\left(\boldsymbol{t}_{1}(\boldsymbol{q} ; \mathbf{y}) \times \boldsymbol{t}_{1}(\boldsymbol{q} ; \mathbf{x})\right)+2\left(\mathbf{g r a d}^{\mathbf{z}} \Phi\right)(\kappa, \boldsymbol{q}(\mathbf{x})-\boldsymbol{q}(\mathbf{y})) \\
& \cdot\left(\left(\partial_{\boldsymbol{q}} \boldsymbol{t}_{1}[\boldsymbol{q}] \boldsymbol{\xi}\right)(\mathbf{y}) \times \boldsymbol{t}_{1}(\boldsymbol{q} ; \mathbf{x})+\boldsymbol{t}_{1}(\boldsymbol{q} ; \mathbf{y})\right. \\
& \left.\times\left(\partial_{\boldsymbol{q}} \boldsymbol{t}_{1}[\boldsymbol{q}] \boldsymbol{\xi}\right)(\mathbf{x})\right), \\
\left(\partial_{\boldsymbol{q}} \mathcal{M}_{2}^{2}[\boldsymbol{q}] \boldsymbol{\xi}\right)(\mathbf{x}, \mathbf{y})= & \left.2\left\{\left(\mathbf{H e s s}^{\mathbf{z}} \Phi\right)(\kappa, \boldsymbol{q}(\mathbf{x})-\boldsymbol{q}(\mathbf{y}))\right](\boldsymbol{\xi}(\mathbf{x})-\boldsymbol{\xi}(\mathbf{y}))\right\} \\
& \cdot\left(\boldsymbol{t}_{2}(\boldsymbol{q} ; \mathbf{y}) \times \boldsymbol{t}_{2}(\boldsymbol{q} ; \mathbf{x})\right)+2\left(\operatorname{grad}^{\mathbf{z}} \Phi\right)(\kappa, \boldsymbol{q}(\mathbf{x})-\boldsymbol{q}(\mathbf{y})) \\
& \cdot\left(\left(\partial_{\boldsymbol{q}} \boldsymbol{t}_{2}[\boldsymbol{q}] \boldsymbol{\xi}\right)(\mathbf{y}) \times \boldsymbol{t}_{1}(\boldsymbol{q} ; \mathbf{x})+\boldsymbol{t}_{2}(\boldsymbol{q} ; \mathbf{y})\right. \\
& \left.\times\left(\partial_{\boldsymbol{q}} \boldsymbol{t}_{1}[\boldsymbol{q}] \boldsymbol{\xi}\right)(\mathbf{x})\right),
\end{aligned}
$$

with

$$
\begin{aligned}
\operatorname{Hess}^{\mathbf{z}} \Phi(\kappa, \mathbf{z}) & =\frac{\Phi(\kappa, \mathbf{z})}{|\mathbf{z}|}\left(i \kappa-\frac{1}{|\mathbf{z}|}\right) \mathrm{I}+\frac{\Phi(\kappa, \mathbf{z})}{|\mathbf{z}|^{2}}\left(-\kappa^{2}-\frac{3 i \kappa}{|\mathbf{z}|}+\frac{3}{|\mathbf{z}|^{2}}\right) \mathbf{z} \otimes \mathbf{z} \\
& =\mathbf{H}_{1}(\kappa, \mathbf{z})+\mathbf{H}_{2}(\kappa, \mathbf{z}) .
\end{aligned}
$$

Proof. The complete proof should be realized in two parts. First we prove that the Fréchet differentiability of the boundary integral operators is obtained by proving the Fréchet differentiability of the kernel. Then we prove that the first Fréchet derivative of the kernel in any direction $\boldsymbol{\xi}$ defines a bounded operator from $\boldsymbol{H}_{\mathrm{div}}^{-\frac{1}{2}}\left(\Gamma_{\mathrm{ref}}\right)$ to itself. The first step and the 
computation of the derivatives have been widely detailed in the papers [10, 11, 42, 43]. Therefore we discuss the second point only.

- Let us prove that the kernels $\partial_{\boldsymbol{q}} \mathcal{M}_{i}^{j}[\boldsymbol{q}] \boldsymbol{\xi}$, for $i, j=1,2$, are weakly singular with $\beta=\alpha$. The terms involving $\mathbf{H}_{2}(\kappa, \mathbf{z})$ are treated in the same way as in remark 3.3 (i) since it involves the scalar products $(\boldsymbol{q}(\mathbf{x})-\boldsymbol{q}(\mathbf{y})) \cdot\left(\boldsymbol{t}_{i}(\boldsymbol{q} ; \mathbf{y}) \times \boldsymbol{t}_{j}(\boldsymbol{q} ; \mathbf{x})\right)$. The matrix $\mathbf{H}_{1}$ behaves as $\|\mathbf{z}\|^{-3}$ when $\|\mathbf{z}\| \rightarrow 0$. It remains to analyze the following sum when $\mathbf{x}, \mathbf{y} \in \Gamma_{\text {ref }}$ and $\|\mathbf{x}-\mathbf{y}\| \rightarrow 0$ :

$$
\begin{aligned}
& (\boldsymbol{\xi}(\mathbf{x})-\boldsymbol{\xi}(\mathbf{y})) \cdot\left(\boldsymbol{t}_{i}(\boldsymbol{q} ; \mathbf{y}) \times \boldsymbol{t}_{j}(\boldsymbol{q} ; \mathbf{x})\right) \\
& \quad+(\boldsymbol{q}(\mathbf{x})-\boldsymbol{q}(\mathbf{y})) \cdot\left(\left(\partial_{\boldsymbol{q}} \boldsymbol{t}_{i}[\boldsymbol{q}] \boldsymbol{\xi}\right)(\mathbf{y}) \times \boldsymbol{t}_{j}(\boldsymbol{q} ; \mathbf{x})+\boldsymbol{t}_{i}(\boldsymbol{q} ; \mathbf{y}) \times\left(\partial_{\boldsymbol{q}} \boldsymbol{t}_{j}[\boldsymbol{q}] \boldsymbol{\xi}\right)(\mathbf{x})\right) .
\end{aligned}
$$

We use

$$
\begin{aligned}
& \boldsymbol{q}(\mathbf{x})-\boldsymbol{q}(\mathbf{y})=\left[\mathrm{D}_{\Gamma_{\mathrm{ref}}} \boldsymbol{q}(\mathbf{x})\right](\mathbf{y}-\mathbf{x})+O\left(\|\mathbf{x}-\mathbf{y}\|^{1+\alpha}\right), \\
& \boldsymbol{\xi}(\mathbf{x})-\boldsymbol{\xi}(\mathbf{y})=\left[\mathrm{D}_{\Gamma_{\mathrm{ref}}} \boldsymbol{\xi}(\mathbf{x})\right](\mathbf{y}-\mathbf{x})+O\left(\|\mathbf{x}-\mathbf{y}\|^{1+\alpha}\right),
\end{aligned}
$$

$\left[\mathrm{D}_{\Gamma_{\text {ref }}} \boldsymbol{\xi}\right]=\left[\mathrm{D}_{\Gamma_{\text {ref }}} \boldsymbol{\xi}\right]\left[\mathrm{D}_{\Gamma_{\text {ref }}} \boldsymbol{q}\right]^{-1}\left[\mathrm{D}_{\Gamma_{\text {ref }}} \boldsymbol{q}\right]$ and $\mathrm{P} a \times b+a \times \mathrm{P} b=\left(\operatorname{Trace}(\mathrm{P}) \mathrm{I}-{ }^{\mathrm{T}} \mathrm{P}\right)(a \times b)$ if $\mathrm{P}$ is 3 by 3 matrix and $a, b$ are two vectors in $\mathbb{R}^{3}$. We obtain

$$
\begin{aligned}
\left(\left[\mathrm{D}_{\Gamma_{\mathrm{ref}}} \boldsymbol{\xi}(\mathbf{x})\right](\mathbf{y}-\mathbf{x})\right) \cdot\left(\boldsymbol{t}_{i}(\boldsymbol{q} ; \mathbf{y}) \times \boldsymbol{t}_{j}(\boldsymbol{q} ; \mathbf{x})\right) \\
\quad+\operatorname{Trace}\left(\left[\mathrm{D}_{\Gamma_{\mathrm{ref}}} \boldsymbol{\xi}(\mathbf{x})\right]\left[\mathrm{D}_{\Gamma_{\mathrm{ref}}} \boldsymbol{q}(\mathbf{x})\right]^{-1}\right)\left(\left[\mathrm{D}_{\Gamma_{\mathrm{ref}}} \boldsymbol{q}(\mathbf{x})\right](\mathbf{y}-\mathbf{x})\right) \cdot\left(\boldsymbol{t}_{i}(\boldsymbol{q} ; \mathbf{y}) \times \boldsymbol{t}_{j}(\boldsymbol{q} ; \mathbf{x})\right) \\
\quad-\left(\left[\mathrm{D}_{\Gamma_{\mathrm{ref}}} \boldsymbol{q}(\mathbf{x})\right](\mathbf{y}-\mathbf{x})\right) \cdot\left({ }^{\mathrm{T}}\left(\left[\mathrm{D}_{\Gamma_{\mathrm{ref}}} \boldsymbol{\xi}(\mathbf{x})\right]\left[\mathrm{D}_{\Gamma_{\mathrm{ref}}} \boldsymbol{q}(\mathbf{x})\right]^{-1}\right)\left(\boldsymbol{t}_{i}(\boldsymbol{q} ; \mathbf{y}) \times \boldsymbol{t}_{j}(\boldsymbol{q} ; \mathbf{x})\right)\right) \\
\quad+\boldsymbol{O}\left(\|\mathbf{x}-\mathbf{y}\|^{1+\alpha}\right) \\
=\operatorname{Trace}\left(\left[\mathrm{D}_{\Gamma_{\mathrm{ref}}} \boldsymbol{\xi}(\mathbf{x})\right]\left[\mathrm{D}_{\Gamma_{\mathrm{ref}}} \boldsymbol{q}(\mathbf{x})\right]^{-1}\right)\left(\left[\mathrm{D}_{\Gamma_{\mathrm{ref}}} \boldsymbol{q}(\mathbf{x})\right](\mathbf{y}-\mathbf{x})\right) \cdot\left(\boldsymbol{t}_{i}(\boldsymbol{q} ; \mathbf{y}) \times \boldsymbol{t}_{j}(\boldsymbol{q} ; \mathbf{x})\right) \\
\quad+\boldsymbol{O}\left(\|\mathbf{x}-\mathbf{y}\|^{1+\alpha}\right) \text { since the sum of the first and third terms vanishes } \\
=\boldsymbol{O}\left(\|\mathbf{x}-\mathbf{y}\|^{1+\alpha}\right) \text { using remark 3.3(i). }
\end{aligned}
$$

We point out that we obtained the usual material derivative results: the term involving Trace $\left(\left[\mathrm{D}_{\Gamma_{\mathrm{ref}}} \xi\right]\left[\mathrm{D}_{\Gamma_{\mathrm{ref}}} \boldsymbol{q}\right]^{-1}\right)$ gives the material derivative of $J_{\boldsymbol{q}}$, that is $J_{\boldsymbol{q}}\left(\operatorname{div}_{\Gamma_{\boldsymbol{q}}}\left(\boldsymbol{\xi} \circ \boldsymbol{q}^{-1}\right)\right) \circ \boldsymbol{q}$, and the one involving ${ }^{\top}\left(\left[\mathrm{D}_{\Gamma_{\mathrm{ref}}} \boldsymbol{\xi}(\mathbf{x})\right]\left[\mathrm{D}_{\Gamma_{\mathrm{ref}}} \boldsymbol{q}(\mathbf{x})\right]^{-1}\right)$ gives the material derivative of $\boldsymbol{n}_{\boldsymbol{q}} \circ \boldsymbol{q}$, that is $-\left(\left[\operatorname{grad}_{\Gamma_{\boldsymbol{q}}} \boldsymbol{\xi} \circ \boldsymbol{q}^{-1}\right] \boldsymbol{n}_{\boldsymbol{q}}\right) \circ \boldsymbol{q}$.

- Let us prove that $\operatorname{div}_{\Gamma_{\text {ref }}} \partial_{\boldsymbol{q}} \widehat{M}_{\kappa}[\boldsymbol{q}, \cdot] \boldsymbol{\xi} \in H^{-\frac{1}{2}}(\Gamma)$. We use the possibility to interchange the differentiation with respect to $\boldsymbol{q}$ and the differentiation with respect to $\mathbf{x}$ because the operator $\operatorname{div}_{\Gamma_{\text {ref }}}$ does not depend on $\boldsymbol{q}$ :

$$
\operatorname{div}_{\Gamma_{\text {ref }}} \partial_{\boldsymbol{q}} \widehat{M}_{\kappa}[\boldsymbol{q}, \cdot] \boldsymbol{\xi}=\partial_{\boldsymbol{q}}\left(\operatorname{div}_{\Gamma_{\mathrm{ref}}} \widehat{M}_{\kappa}\right)[\boldsymbol{q}, \cdot] \boldsymbol{\xi}
$$

Using (3.3) and (3.4) we find that the kernel of $\operatorname{div}_{\Gamma_{\text {ref }}} \widehat{M}_{\kappa}(\boldsymbol{q}, \boldsymbol{j})$ is

$$
\begin{aligned}
& -\kappa^{2} \Phi(\kappa, \boldsymbol{q}(\mathbf{x})-\boldsymbol{q}(\mathbf{y})) J_{\boldsymbol{q}}(\mathbf{x}) \boldsymbol{n}_{\boldsymbol{q}}(\boldsymbol{q}(\mathbf{x})) \cdot \mathcal{P}_{\boldsymbol{q}}^{-1} \boldsymbol{j}(\mathbf{y}) \\
& \quad-J_{\boldsymbol{q}}(\mathbf{x}) \boldsymbol{n}_{\boldsymbol{q}}(\boldsymbol{q}(\mathbf{x})) \cdot \operatorname{grad}^{\boldsymbol{q}(\mathbf{x})} \Phi(\kappa, \boldsymbol{q}(\mathbf{x})-\boldsymbol{q}(\mathbf{y})) \operatorname{div}_{\Gamma_{\mathrm{ref}}} \boldsymbol{j}(\mathbf{y}) .
\end{aligned}
$$

The kernel of the first term is weakly singular with $\beta=1$. We have $\operatorname{div}_{\Gamma_{\mathrm{ref}}} \boldsymbol{j} \in H^{-\frac{1}{2}}\left(\Gamma_{\mathrm{ref}}\right)$ independent of $\boldsymbol{q}$ and the kernel of the second term is weakly singular with $\beta=\alpha$. By differentiation with respect to $\boldsymbol{q}$ we obtain the same results (we refer to [42]). 
For any $\boldsymbol{j} \in \boldsymbol{H}_{\text {div }}^{-\frac{1}{2}}\left(\Gamma_{\text {ref }}\right)$, we set $\widehat{C}_{\kappa}(\boldsymbol{q}, \boldsymbol{j})=\mathcal{P}_{\boldsymbol{q}} C_{\kappa}(\boldsymbol{q}) \mathcal{P}_{\boldsymbol{q}}^{-1} \boldsymbol{j}$ and we have

$$
\begin{aligned}
\widehat{C}_{\kappa}(\boldsymbol{q}, \boldsymbol{j})(\mathbf{x})= & \kappa J_{q}(\mathbf{x})\left[\mathrm{D}_{\Gamma_{\mathrm{ref}}} \boldsymbol{q}(\mathbf{x})\right]^{-1} \int_{\Gamma_{\mathrm{ref}}} \boldsymbol{n}_{\boldsymbol{q}}(\boldsymbol{q}(\mathbf{x})) \\
& \times\left\{2 \Phi(\kappa, \boldsymbol{q}(\mathbf{x})-\boldsymbol{q}(\mathbf{y}))\left[\mathrm{D}_{\Gamma_{\mathrm{ref}}} \boldsymbol{q}(\mathbf{y})\right] \boldsymbol{j}(\mathbf{y})\right\} d s(\mathbf{y}) \\
& -\frac{1}{\kappa}\left(\operatorname{curl}_{\Gamma_{\mathrm{ref}}} \int_{\Gamma_{\mathrm{ref}}} 2 \Phi(\kappa, \boldsymbol{q}(\cdot)-\boldsymbol{q}(\mathbf{y})) \operatorname{div}_{\Gamma_{\mathrm{ref}}} \boldsymbol{j}(\mathbf{y}) d s(\mathbf{y})\right)(\mathbf{x})
\end{aligned}
$$

From (3.2) and (3.3), this can be rewritten

$$
\begin{aligned}
\widehat{C}_{\kappa}(\boldsymbol{q}, \boldsymbol{j})(\mathbf{x})= & \kappa \boldsymbol{n}(\mathbf{x}) \times \int_{\Gamma_{\mathrm{ref}}}{ }^{\mathrm{T}}\left[\mathrm{D}_{\Gamma_{\mathrm{ref}}} \boldsymbol{q}(\mathbf{x})\right]\left\{2 \Phi(\kappa, \boldsymbol{q}(\mathbf{x})-\boldsymbol{q}(\mathbf{y}))\left[\mathrm{D}_{\Gamma_{\mathrm{ref}}} \boldsymbol{q}(\mathbf{y})\right] \boldsymbol{j}(\mathbf{y})\right\} d s(\mathbf{y}) \\
& -\frac{1}{\kappa}\left(\operatorname{curl}_{\Gamma_{\mathrm{ref}}} \int_{\Gamma_{\mathrm{ref}}} 2 \Phi(\kappa, \boldsymbol{q}(\cdot)-\boldsymbol{q}(\mathbf{y})) \operatorname{div}_{\Gamma_{\mathrm{ref}}} \boldsymbol{j}(\mathbf{y}) d s(\mathbf{y})\right)(\mathbf{x}) \\
= & \kappa \boldsymbol{e}^{1}(\mathbf{x}) \int_{\Gamma_{\mathrm{ref}}}\left\{\mathcal{C}_{1}^{1}(\boldsymbol{q} ; \mathbf{x}, \mathbf{y})\left(\boldsymbol{j}(\mathbf{y}) \cdot \boldsymbol{e}_{1}(\mathbf{y})\right)+\mathcal{C}_{2}^{1}(\boldsymbol{q} ; \mathbf{x}, \mathbf{y})\left(\boldsymbol{j}(\mathbf{y}) \cdot \boldsymbol{e}_{2}(\mathbf{y})\right)\right\} d s(\mathbf{y}) \\
& +\kappa \boldsymbol{e}^{2}(\mathbf{x}) \int_{\Gamma_{\mathrm{ref}}}\left\{\mathcal{C}_{1}^{2}(\boldsymbol{q} ; \mathbf{x}, \mathbf{y})\left(\boldsymbol{j}(\mathbf{y}) \cdot \boldsymbol{e}_{1}(\mathbf{y})\right)+\mathcal{C}_{2}^{2}(\boldsymbol{q} ; \mathbf{x}, \mathbf{y})\left(\boldsymbol{j}(\mathbf{y}) \cdot \boldsymbol{e}_{2}(\mathbf{y})\right)\right\} d s(\mathbf{y}) \\
& -\frac{1}{\kappa}\left(\operatorname{curl}_{\Gamma_{\mathrm{ref}}} \int_{\Gamma_{\mathrm{ref}}} 2 \Phi(\kappa, \boldsymbol{q}(\cdot)-\boldsymbol{q}(\mathbf{y})) \operatorname{div}_{\Gamma_{\mathrm{ref}}} \boldsymbol{j}(\mathbf{y}) d s(\mathbf{y})\right)(\mathbf{x})
\end{aligned}
$$

where

$$
\begin{aligned}
& \mathcal{C}_{1}^{1}(\boldsymbol{q} ; \mathbf{x}, \mathbf{y})=-2 \Phi(\kappa, \boldsymbol{q}(\mathbf{x})-\boldsymbol{q}(\mathbf{y}))\left(\boldsymbol{t}_{1}(\boldsymbol{q} ; \mathbf{y}) \cdot \boldsymbol{t}_{2}(\boldsymbol{q} ; \mathbf{x})\right) \\
& \mathcal{C}_{2}^{1}(\boldsymbol{q} ; \mathbf{x}, \mathbf{y})=-2 \Phi(\kappa, \boldsymbol{q}(\mathbf{x})-\boldsymbol{q}(\mathbf{y}))\left(\boldsymbol{t}_{2}(\boldsymbol{q} ; \mathbf{y}) \cdot \boldsymbol{t}_{2}(\boldsymbol{q} ; \mathbf{x})\right) \\
& \mathcal{C}_{1}^{2}(\boldsymbol{q} ; \mathbf{x}, \mathbf{y})=2 \Phi(\kappa, \boldsymbol{q}(\mathbf{x})-\boldsymbol{q}(\mathbf{y}))\left(\boldsymbol{t}_{1}(\boldsymbol{q} ; \mathbf{y}) \cdot \boldsymbol{t}_{1}(\boldsymbol{q} ; \mathbf{x})\right) \\
& \mathcal{C}_{2}^{2}(\boldsymbol{q} ; \mathbf{x}, \mathbf{y})=2 \Phi(\kappa, \boldsymbol{q}(\mathbf{x})-\boldsymbol{q}(\mathbf{y}))\left(\boldsymbol{t}_{2}(\boldsymbol{q} ; \mathbf{y}) \cdot \boldsymbol{t}_{1}(\boldsymbol{q} ; \mathbf{x})\right)
\end{aligned}
$$

Theorem 3.5. The mapping $\widehat{C}_{k}: \mathcal{Q}_{a d} \rightarrow \mathscr{L}\left(\boldsymbol{H}_{\mathrm{div}}^{-\frac{1}{2}}\left(\Gamma_{\mathrm{ref}}\right)\right)$ is Fréchet differentiable at all $\boldsymbol{q} \in \mathcal{Q}_{a d}$ and the first derivatives at $\boldsymbol{q}$ in the direction $\boldsymbol{\xi} \in \mathscr{C}^{1, \alpha}\left(\Gamma_{\text {ref }}\right)$ is a bounded linear integral operator in $\mathscr{L}\left(\boldsymbol{H}_{\mathrm{div}}^{-\frac{1}{2}}\left(\Gamma_{\mathrm{ref}}\right)\right)$ defined for $\boldsymbol{j} \in \boldsymbol{H}_{\mathrm{div}}^{-\frac{1}{2}}\left(\Gamma_{\mathrm{ref}}\right)$ by

$$
\begin{aligned}
\left(\partial_{\boldsymbol{q}} \widehat{C}_{\kappa}[\boldsymbol{q}, \boldsymbol{j}] \boldsymbol{\xi}\right)(\mathbf{x})= & \kappa \boldsymbol{e}^{1}(\mathbf{x}) \int_{\Gamma_{\mathrm{ref}}}\left\{\left(\partial_{\boldsymbol{q}} \mathcal{C}_{1}^{1}[\boldsymbol{q}] \boldsymbol{\xi}\right)(\mathbf{x}, \mathbf{y})\left(\boldsymbol{j}(\mathbf{y}) \cdot \boldsymbol{e}_{1}(\mathbf{y})\right)\right. \\
& \left.+\left(\partial_{\boldsymbol{q}} \mathcal{C}_{2}^{1}[\boldsymbol{q}] \boldsymbol{\xi}\right)(\mathbf{x}, \mathbf{y})\left(\boldsymbol{j}(\mathbf{y}) \cdot \boldsymbol{e}_{2}(\mathbf{y})\right)\right\} d s(\mathbf{y}) \\
& +\kappa \boldsymbol{e}^{2}(\mathbf{x}) \int_{\Gamma_{\mathrm{ref}}}\left\{\left(\partial_{\boldsymbol{q}} \mathcal{C}_{1}^{2}[\boldsymbol{q}] \boldsymbol{\xi}\right)(\mathbf{x}, \mathbf{y})\left(\boldsymbol{j}(\mathbf{y}) \cdot \boldsymbol{e}_{1}(\mathbf{y})\right)\right. \\
& \left.+\left(\partial_{\boldsymbol{q}} \mathcal{C}_{2}^{2}[\boldsymbol{q}] \boldsymbol{\xi}\right)(\mathbf{x}, \mathbf{y})\left(\boldsymbol{j}(\mathbf{y}) \cdot \boldsymbol{e}_{2}(\mathbf{y})\right)\right\} d s(\mathbf{y}) \\
& -\frac{1}{\kappa}\left(\operatorname { c u r l } _ { \Gamma _ { \mathrm { ref } } } \int _ { \Gamma _ { \mathrm { ref } } } \left\{\left(2 \operatorname{grad}^{\mathbf{z}} \Phi\right)(\kappa, \boldsymbol{q}(\cdot)-\boldsymbol{q}(\mathbf{y}))\right.\right. \\
& \left.\left.\cdot(\boldsymbol{\xi}(\cdot)-\boldsymbol{\xi}(\mathbf{y})) \operatorname{div}_{\Gamma_{\mathrm{ref}}} \boldsymbol{j}(\mathbf{y})\right\} d s(\mathbf{y})\right)(\mathbf{x}),
\end{aligned}
$$


where

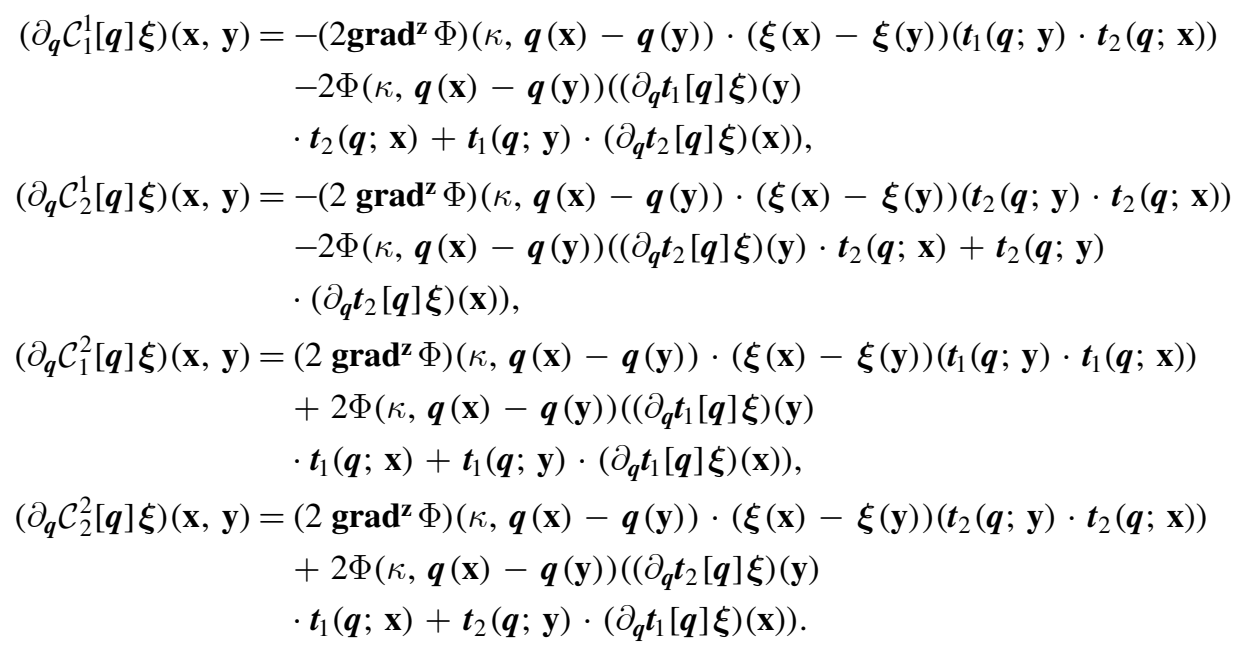

Proof. To obtain the expression of the material derivatives we use again the possibility to interchange the differentiation with respect to $\boldsymbol{q}$ with the surface differential operators and the integral sign on $\Gamma_{\text {ref }}$. Using the Taylor-Young expansions of $\boldsymbol{q}$ and $\boldsymbol{\xi}$ given in remark 3.3 it is easy to prove that the kernels of the derivatives $\partial_{q} \mathcal{C}_{k}^{\ell}[q] \xi$, for $\ell, k=1,2$, are weakly singular with $\beta=1$. Employing the chain rule, we obtain the boundedness of the first derivative from $\boldsymbol{H}_{\mathrm{div}}^{-\frac{1}{2}}\left(\Gamma_{\mathrm{ref}}\right)$ to $\boldsymbol{H}_{\mathrm{t}}^{-\frac{1}{2}}\left(\Gamma_{\mathrm{ref}}\right)$. Applying the operator $\operatorname{div}_{\Gamma_{\mathrm{ref}}}$ to $\partial_{\boldsymbol{q}} \widehat{C}_{\kappa}[\boldsymbol{q}, \boldsymbol{j}] \boldsymbol{\xi}$, the third term vanishes so that the operator $\operatorname{div}_{\Gamma_{\text {ref }}}\left(\partial_{\boldsymbol{q}} \widehat{C}_{\kappa}[\boldsymbol{q}, \cdot] \boldsymbol{\xi}\right)=\partial_{\boldsymbol{q}}\left(\operatorname{div}_{\Gamma_{\text {ref }}} \widehat{C}_{\kappa}\right)[\boldsymbol{q}, \cdot] \boldsymbol{\xi}$ is bounded from $\boldsymbol{H}_{\mathrm{div}}^{-\frac{1}{2}}\left(\Gamma_{\mathrm{ref}}\right)$ to $H^{-\frac{1}{2}}\left(\Gamma_{\text {ref }}\right)$.

We set $\widehat{\mathscr{S}}_{\kappa}^{\infty}(\boldsymbol{q}, \boldsymbol{j})=\mathscr{S}_{\kappa}^{\infty}(\boldsymbol{q}) \mathcal{P}_{\boldsymbol{q}}^{-1} \boldsymbol{j}$ and $\widehat{\mathscr{D}}_{\kappa}^{\infty}(\boldsymbol{q}, \boldsymbol{j})=\mathscr{D}_{\kappa}^{\infty}(\boldsymbol{q}) \mathcal{P}_{\boldsymbol{q}}^{-1} \boldsymbol{j}$ and we have

$$
\begin{aligned}
\widehat{\mathscr{S}}_{\kappa}^{\infty}(\boldsymbol{q}, \boldsymbol{j})(\widehat{\mathbf{x}})= & \frac{\kappa}{4 \pi}\left(\widehat{\mathbf{x}} \times \int_{\Gamma_{\mathrm{ref}}} e^{-i \kappa \hat{\mathbf{x}} \cdot \boldsymbol{q}(\mathbf{y})}\left\{\left(\boldsymbol{j}(\mathbf{y}) \cdot \boldsymbol{e}_{1}(\mathbf{y})\right) \boldsymbol{t}_{1}(\boldsymbol{q} ; \mathbf{y})\right.\right. \\
& \left.\left.+\left(\boldsymbol{j}(\mathbf{y}) \cdot \boldsymbol{e}_{2}(\mathbf{y})\right) \boldsymbol{t}_{2}(\boldsymbol{q} ; \mathbf{y})\right\} d s(\mathbf{y})\right) \times \widehat{\mathbf{x}},
\end{aligned}
$$

and

$$
\begin{aligned}
\widehat{\mathscr{D}}_{\kappa}^{\infty}(\boldsymbol{q}, \boldsymbol{j})(\widehat{\mathbf{x}})= & \frac{i \kappa}{4 \pi} \widehat{\mathbf{x}} \int_{\Gamma_{\mathrm{ref}}} e^{-i \kappa \widehat{\mathbf{x}} \cdot \boldsymbol{q}(\mathbf{y})}\left\{\left(\boldsymbol{j}(\mathbf{y}) \cdot \boldsymbol{e}_{1}(\mathbf{y})\right) \boldsymbol{t}_{1}(\boldsymbol{q} ; \mathbf{y})\right. \\
& \left.+\left(\boldsymbol{j}(\mathbf{y}) \cdot \boldsymbol{e}_{2}(\mathbf{y})\right) \boldsymbol{t}_{2}(\boldsymbol{q} ; \mathbf{y})\right\} d s(\mathbf{y})
\end{aligned}
$$

Theorem 3.6. The mappings $\widehat{\mathscr{S}}_{\kappa}^{\infty}, \widehat{\mathscr{D}}_{\kappa}^{\infty}: \mathcal{Q}_{a d} \rightarrow \mathscr{L}\left(\boldsymbol{L}_{\mathrm{t}}^{2}\left(\Gamma_{\mathrm{ref}}\right), \boldsymbol{L}_{\mathrm{t}}^{2}\left(\mathbb{S}^{2}\right)\right)$ are Fréchet differentiable at all $\boldsymbol{q} \in \mathcal{Q}_{\text {ad }}$ and the first derivatives at $\boldsymbol{q}$ in the direction $\boldsymbol{\xi} \in \mathscr{C}^{1, \alpha}\left(\Gamma_{\mathrm{ref}}, \mathbb{R}^{3}\right)$ are bounded linear integral operators in $\mathscr{L}\left(\boldsymbol{L}_{\mathrm{t}}^{2}\left(\Gamma_{\mathrm{ref}}\right), \boldsymbol{L}_{\mathrm{t}}^{2}\left(\mathbb{S}^{2}\right)\right)$ defined for $\boldsymbol{j} \in \boldsymbol{L}_{\mathrm{t}}^{2}\left(\Gamma_{\mathrm{ref}}\right)$ by 


$$
\begin{aligned}
\left(\partial_{\boldsymbol{q}} \widehat{\mathscr{S}}_{\kappa}^{\infty}[\boldsymbol{q}, \boldsymbol{j}] \boldsymbol{\xi}\right)(\widehat{\mathbf{x}})= & \frac{\kappa}{4 \pi}\left(\widehat{\mathbf{x}} \times \int_{\Gamma_{\mathrm{ref}}}\left\{\left(\boldsymbol{j}(\mathbf{y}) \cdot \boldsymbol{e}_{1}(\mathbf{y})\right)\left(\partial_{\boldsymbol{q}} \mathcal{W}_{1}^{\infty}[\boldsymbol{q}] \boldsymbol{\xi}\right)(\widehat{\mathbf{x}}, \mathbf{y})\right.\right. \\
& \left.\left.+\left(\boldsymbol{j}(\mathbf{y}) \cdot \boldsymbol{e}_{2}(\mathbf{y})\right)\left(\partial_{\boldsymbol{q}} \mathcal{W}_{2}^{\infty}[\boldsymbol{q}] \boldsymbol{\xi}\right)(\widehat{\mathbf{x}}, \mathbf{y})\right\} d s(\mathbf{y})\right) \times \widehat{\mathbf{x}} \\
\left(\partial_{\boldsymbol{q}} \widehat{\mathscr{D}}_{\kappa}^{\infty}[\boldsymbol{q}, \boldsymbol{j}] \boldsymbol{\xi}\right)(\widehat{\mathbf{x}})= & \frac{i \kappa}{4 \pi} \widehat{\mathbf{x}} \times \int_{\Gamma_{\mathrm{ref}}}\left\{\left(\boldsymbol{j}(\mathbf{y}) \cdot \boldsymbol{e}_{1}(\mathbf{y})\right)\left(\partial_{\boldsymbol{q}} \mathcal{W}_{1}^{\infty}[\boldsymbol{q}] \boldsymbol{\xi}\right)(\widehat{\mathbf{x}}, \mathbf{y})\right. \\
& \left.+\left(\boldsymbol{j}(\mathbf{y}) \cdot \boldsymbol{e}_{2}(\mathbf{y})\right)\left(\partial_{\boldsymbol{q}} \mathcal{W}_{2}^{\infty}[\boldsymbol{q}] \boldsymbol{\xi}\right)(\widehat{\mathbf{x}}, \mathbf{y})\right\} d s(\mathbf{y}),
\end{aligned}
$$

where

$$
\begin{aligned}
& \left(\partial_{q} \mathcal{W}_{1}^{\infty}[\boldsymbol{q}] \boldsymbol{\xi}\right)(\widehat{\mathbf{x}}, \mathbf{y})=e^{-i \kappa \hat{\mathbf{x}} \cdot \boldsymbol{q}(\mathbf{y})}\left\{-i \kappa(\widehat{\mathbf{x}} \cdot \boldsymbol{\xi}(\mathbf{y})) \boldsymbol{t}_{1}(\boldsymbol{q} ; \mathbf{y})+\left(\partial_{q} \boldsymbol{t}_{1}[\boldsymbol{q}] \boldsymbol{\xi}\right)(\mathbf{y})\right\} \\
& \left(\partial_{\boldsymbol{q}} \mathcal{W}_{2}^{\infty}[\boldsymbol{q}] \boldsymbol{\xi}\right)(\widehat{\mathbf{x}}, \mathbf{y})=e^{-i \kappa \hat{\mathbf{x}} \cdot \boldsymbol{q}(\mathbf{y})}\left\{-i \kappa(\widehat{\mathbf{x}} \cdot \boldsymbol{\xi}(\mathbf{y})) \boldsymbol{t}_{2}(\boldsymbol{q} ; \mathbf{y})+\left(\partial_{\boldsymbol{q}} \boldsymbol{t}_{2}[\boldsymbol{q}] \boldsymbol{\xi}\right)(\mathbf{y})\right\}
\end{aligned}
$$

Proof. The proof is established in [11]. The use of the Piola transform instead of the Hodge decomposition does not pose additional issues.

Hence with the aid of theorems 3.3-3.5 we found Fréchet derivatives of the following operators defined on $\boldsymbol{H}_{\mathrm{div}}^{-\frac{1}{2}}\left(\Gamma_{\mathrm{ref}}\right) \cap \boldsymbol{L}_{\mathrm{t}}^{2}\left(\Gamma_{\mathrm{ref}}\right): \widehat{\mathrm{I}}_{o p}(\boldsymbol{q}, \cdot)=\mathrm{I}+\widehat{M}_{\kappa}(\boldsymbol{q}, \cdot)+i \eta \widehat{\boldsymbol{C}}_{\kappa}(\boldsymbol{q}, \cdot) \widehat{\boldsymbol{\Lambda}}$ and $\widehat{\mathscr{F}}^{\infty}(\boldsymbol{q}, \cdot)=\widehat{\mathscr{D}}_{\kappa}^{\infty}(\boldsymbol{q}, \cdot)+i \eta \widehat{\mathscr{S}}_{\kappa}^{\infty}(\boldsymbol{q}, \cdot) \widehat{\boldsymbol{\Lambda}}$. They are given by $\partial_{\boldsymbol{q}} \widehat{\mathrm{I}}_{o p}[\boldsymbol{q}, \boldsymbol{j}]=\partial_{\boldsymbol{q}} \widehat{M}_{\kappa}[\boldsymbol{q}, \boldsymbol{j}]$ $+i \eta \partial_{\boldsymbol{q}} \widehat{C}_{\kappa}[\boldsymbol{q}, \boldsymbol{j}] \widehat{\boldsymbol{\Lambda}}$ and $\partial_{\boldsymbol{q}} \widehat{\mathscr{F}}^{\infty}[\boldsymbol{q}, \boldsymbol{j}]=\partial_{\boldsymbol{q}} \widehat{\mathscr{D}}_{\kappa}^{\infty}[\boldsymbol{q}, \boldsymbol{j}]+i \eta \partial_{\boldsymbol{q}} \widehat{\mathscr{S}}_{\kappa}^{\infty}[\boldsymbol{q}, \boldsymbol{j}] \widehat{\boldsymbol{\Lambda}}$, correspondingly.

We have, $k=1, \ldots, m, \boldsymbol{f}_{k}(\boldsymbol{q})=-2 \mathcal{P}_{\boldsymbol{q}}\left(\boldsymbol{n}_{\boldsymbol{q}} \times \boldsymbol{E}_{k, \mid \Gamma_{\boldsymbol{q}}}^{i n c}\right)$. By the definition of the incident plane wave and (2.8) we obtain

$$
\boldsymbol{f}_{k}(\boldsymbol{q})(\mathbf{x})=-2 J_{\boldsymbol{q}}\left[\mathrm{D}_{\Gamma_{\mathrm{ref}}} \boldsymbol{q}(\mathbf{x})\right]^{-1}\left(\boldsymbol{n}_{\boldsymbol{q}}(\mathbf{x}) \times \boldsymbol{p}_{k} e^{i \kappa \boldsymbol{q}(\mathbf{x}) \cdot \boldsymbol{d}_{k}}\right) .
$$

Since $\left[\mathrm{D}_{\Gamma_{\text {ref }}} \boldsymbol{q}\right]^{-1}=\boldsymbol{e}_{1} \otimes \boldsymbol{t}^{1}(\boldsymbol{q})+\boldsymbol{e}_{2} \otimes \boldsymbol{t}^{2}(\boldsymbol{q})$ the function $\boldsymbol{f}_{k}$ can be rewritten as following

$$
\boldsymbol{f}_{k}(\boldsymbol{q})(\mathbf{x})=2 e^{i \kappa \boldsymbol{q}(\mathbf{x}) \cdot \boldsymbol{d}_{k}}\left(\boldsymbol{e}_{1}(\mathbf{x})\left(\boldsymbol{p}_{k} \cdot \boldsymbol{t}_{2}(\boldsymbol{q} ; \mathbf{x})\right)-\boldsymbol{e}_{2}(\mathbf{x})\left(\boldsymbol{p}_{k} \cdot \boldsymbol{t}_{1}(\boldsymbol{q} ; \mathbf{x})\right)\right)
$$

Using the chain rule we obtain the following result.

Proposition 3.7. The mapping $\boldsymbol{f}_{k}: \boldsymbol{q} \in \mathcal{Q}_{a d} \rightarrow \boldsymbol{f}(\boldsymbol{q}) \in \boldsymbol{H}_{\mathrm{div}}^{-\frac{1}{2}}\left(\Gamma_{\mathrm{ref}}\right)$, is Fréchet differentiable at all $\boldsymbol{q} \in \mathcal{Q}_{\text {ad }}$ and the first derivatives at $\boldsymbol{q}$ in the direction $\boldsymbol{\xi} \in \mathscr{C}^{1, \alpha}\left(\Gamma_{\text {ref }}, \mathbb{R}^{3}\right)$ is given by

$$
\begin{aligned}
\left(\partial_{\boldsymbol{q}} \boldsymbol{f}_{k}[\boldsymbol{q}] \boldsymbol{\xi}\right)(\mathbf{x})= & \boldsymbol{f}_{k}(\boldsymbol{q})(\mathbf{x}) i \kappa \boldsymbol{\xi}(\mathbf{x}) \cdot \boldsymbol{d}_{k} \\
& +2 e^{i \kappa \boldsymbol{q}(\mathbf{x}) \cdot \boldsymbol{d}_{k}}\left(\boldsymbol{e}_{1}(\mathbf{x})\left(\boldsymbol{p}_{k} \cdot\left(\partial_{\boldsymbol{q}} \boldsymbol{t}_{2}[\boldsymbol{q}] \boldsymbol{\xi}\right)(\mathbf{x})\right)-\boldsymbol{e}_{2}(\mathbf{x})\left(\boldsymbol{p}_{k} \cdot\left(\partial_{\boldsymbol{q}} \boldsymbol{t}_{1}[\boldsymbol{q}] \boldsymbol{\xi}\right)(\mathbf{x})\right)\right) .
\end{aligned}
$$

\section{Numerical implementation}

To solve the parametrized system of boundary integral equation with smooth and weakly singular kernels we employ a high order spectral algorithm, which was recently introduced by Le Louër [23, 33]. The idea of the method originates from Atkinson's work [2], where he suggested a Galerkin method for the Laplace equation. Later on, Wienert [45] has developed a Nyström method for problems which has been further developed by Ganesh, Graham, Sloan $[15,18]$ into fully discrete Galerkin type method. By combining the spectral method [15] and the hybrid spectral method [16, 17] Le Louër [33] introduced a high order spectral algorithm for the Maxwell's equations, which we employ also in this work.

We assume $\Gamma_{\text {ref }}=\mathbb{S}^{2}$ and consider special parametrizations

$$
\boldsymbol{q}=\mathcal{R} r \quad \text { with } \quad \mathcal{R} r(\widehat{\mathbf{x}}):=r(\widehat{\mathbf{x}}) \widehat{\mathbf{x}}, \widehat{\mathbf{x}} \in \mathbb{S}^{2},
$$


with a function $r: \mathbb{S}^{2} \rightarrow(0, \infty)$ uniquely determined by $\Gamma$. The set of admissible parametrizations is defined as $\mathcal{Q}_{\text {star }}:=\left\{r \in H^{s}\left(\mathbb{S}^{2}, \mathbb{R}\right) ; r>0\right\}, \mathcal{R}\left(\mathcal{Q}_{\text {star }}\right) \subset \mathcal{Q}_{a d}$. The parameterized form of the operator $\mathrm{A}_{k}$ in $(2.10 b)$ is an operator $\mathrm{A}_{k, \text { star }}: \boldsymbol{H}_{\mathrm{div}}^{-\frac{1}{2}}\left(\mathbb{S}^{2}\right) \times H^{s}\left(\mathbb{S}^{2}, \mathbb{R}\right) \rightarrow \boldsymbol{H}_{\mathrm{div}}^{-\frac{1}{2}}\left(\mathbb{S}^{2}\right) \times \boldsymbol{L}_{\mathrm{t}}^{2}\left(\mathbb{S}^{2}\right)$ defined by $\mathrm{A}_{k, \text { star }}=\mathrm{A}_{k} \circ \operatorname{diag}(\mathrm{I}, \mathcal{R})$.

The implementation of the operators $\widehat{M}_{\kappa}, \widehat{C}_{\kappa}$ is already discussed in [23, 33]. Here, we focus on the operator $\partial_{\boldsymbol{q}} \widehat{M}_{\kappa}$. For brevity we use the notation $\boldsymbol{q}_{\widehat{\mathbf{x}}}:=\boldsymbol{q}(\widehat{\mathbf{x}})$. To split off the singularities in the kernels of integral operators we introduce the functions:

$\mathcal{S}_{1}^{\kappa}(\boldsymbol{q} ; \widehat{\mathbf{x}}, \widehat{\mathbf{y}})=\frac{1}{2 \pi} \cos \left(\kappa\left|\boldsymbol{q}_{\widehat{\mathbf{x}}}-\boldsymbol{q}_{\widehat{\mathbf{y}}}\right|\right), \mathcal{S}_{2}^{\kappa}(\boldsymbol{q} ; \widehat{\mathbf{x}}, \widehat{\mathbf{y}})=\frac{1}{2 \pi} \begin{cases}\frac{\sin \left(\kappa\left|\boldsymbol{q}_{\widehat{\mathbf{x}}}-\boldsymbol{q}_{\hat{\mathbf{y}}}\right|\right)}{\left|\boldsymbol{q}_{\widehat{\mathbf{x}}}-\boldsymbol{q}_{\hat{\mathbf{y}}}\right|}, & \widehat{\mathbf{x}} \neq \hat{\mathbf{y}}, \\ \kappa, & \widehat{\mathbf{x}}=\widehat{\mathbf{y}} .\end{cases}$

The operator $\partial_{\boldsymbol{q}} \widehat{M}_{\kappa}\left[\boldsymbol{q}, \boldsymbol{j}_{\boldsymbol{s}}\right]$ can then be rewritten as

$$
\begin{aligned}
\left(\partial_{\boldsymbol{q}} \widehat{M}_{\kappa}\left[\boldsymbol{q}, \boldsymbol{j}_{\boldsymbol{s}}\right] \boldsymbol{\xi}\right)(\widehat{\mathbf{x}})= & \sum_{l=1}^{2}(-1)^{l} \boldsymbol{e}^{l}(\widehat{\mathbf{x}}) \sum_{l^{\prime}=1}^{2} \int_{\mathbb{S}^{2}} \frac{R(\boldsymbol{q} ; \widehat{\mathbf{x}}, \widehat{\mathbf{y}})}{|\widehat{\mathbf{x}}-\widehat{\mathbf{y}}|} \mathrm{s} \mathcal{M}_{\kappa, l^{\prime}}^{l}(\boldsymbol{\xi} ; \boldsymbol{q}, \widehat{\mathbf{x}}, \widehat{\mathbf{y}}) \boldsymbol{j}_{s}^{\boldsymbol{l}^{\prime}}(\widehat{\mathbf{y}}) d s(\widehat{\mathbf{y}}) \\
& +i \sum_{l=1}^{2}(-1)^{l} \boldsymbol{e}^{l}(\widehat{\mathbf{x}}) \sum_{l^{\prime}=1}^{2} \int_{\mathbb{S}^{2}} \mathrm{r}^{\mathcal{M}_{\kappa, l^{\prime}}}(\boldsymbol{\xi} ; \boldsymbol{q}, \widehat{\mathbf{x}}, \widehat{\mathbf{y}}) \boldsymbol{j}_{s}^{l^{\prime}}(\widehat{\mathbf{y}}) d s(\widehat{\mathbf{y}})
\end{aligned}
$$

Here $R(\boldsymbol{q} ; \widehat{\mathbf{x}}, \widehat{\mathbf{y}})=|\widehat{\mathbf{x}}-\widehat{\mathbf{y}}| /\left|\boldsymbol{q}_{\widehat{\mathbf{x}}}-\boldsymbol{q}_{\widehat{\mathbf{y}}}\right|, \quad \boldsymbol{j}_{\boldsymbol{s}}^{\iota^{\prime}}(\widehat{\mathbf{y}})=\left(\boldsymbol{j}_{\boldsymbol{s}}(\widehat{\mathbf{y}}) \cdot \boldsymbol{e}_{l^{\prime}}(\widehat{\mathbf{y}})\right)$,

$$
\begin{aligned}
& \mathrm{s} \mathcal{M}_{\kappa, l^{\prime}}^{l}(\boldsymbol{\xi} ; \boldsymbol{q}, \widehat{\mathbf{x}}, \widehat{\mathbf{y}})=\left(\frac{3 \mathcal{S}_{1}^{\kappa}(\boldsymbol{q} ; \widehat{\mathbf{x}}, \widehat{\mathbf{y}})}{\left|\boldsymbol{q}_{\widehat{\mathbf{x}}}-\boldsymbol{q}_{\widehat{\mathbf{y}}}\right|^{2}}+3 \kappa \mathcal{S}_{2}^{\kappa}(\boldsymbol{q} ; \widehat{\mathbf{x}}, \widehat{\mathbf{y}})-\kappa^{2} \mathcal{S}_{1}^{\kappa}(\boldsymbol{q} ; \widehat{\mathbf{x}}, \widehat{\mathbf{y}})\right) \mathcal{W}_{l^{\prime}}^{l}(\boldsymbol{\xi} ; \boldsymbol{q}, \widehat{\mathbf{x}}, \widehat{\mathbf{y}}) \\
&-\left(\frac{\mathcal{S}_{1}^{\kappa}(\boldsymbol{q} ; \widehat{\mathbf{x}}, \widehat{\mathbf{y}})}{\left|\boldsymbol{q}_{\widehat{\mathbf{x}}}-\boldsymbol{q}_{\widehat{\mathbf{y}}}\right|^{2}}+\kappa \mathcal{S}_{2}^{\kappa}(\boldsymbol{q} ; \widehat{\mathbf{x}}, \widehat{\mathbf{y}})\right) \mathcal{V}_{l^{\prime}}^{l}(\boldsymbol{\xi} ; \boldsymbol{q}, \widehat{\mathbf{x}}, \widehat{\mathbf{y}}), \\
&{ }^{r} \mathcal{M}_{\kappa, l^{\prime}}^{l}(\boldsymbol{\xi} ; \boldsymbol{q}, \widehat{\mathbf{x}}, \widehat{\mathbf{y}})=\left(\frac{-3 \kappa \mathcal{S}_{1}^{\kappa}(\boldsymbol{q} ; \widehat{\mathbf{x}}, \widehat{\mathbf{y}})+3 \mathcal{S}_{2}^{\kappa}(\boldsymbol{q} ; \widehat{\mathbf{x}}, \widehat{\mathbf{y}})}{\left|\boldsymbol{q}_{\widehat{\mathbf{x}}}-\boldsymbol{q}_{\widehat{\mathbf{y}}}\right|^{2}}-\kappa^{2} \mathcal{S}_{2}^{\kappa}(\boldsymbol{q} ; \widehat{\mathbf{x}}, \widehat{\mathbf{y}})\right) \mathcal{W}_{l^{\prime}}^{l}(\boldsymbol{\xi} ; \boldsymbol{q}, \widehat{\mathbf{x}}, \widehat{\mathbf{y}}) \\
&+\frac{\kappa \mathcal{S}_{1}^{\kappa}(\boldsymbol{q} ; \widehat{\mathbf{x}}, \widehat{\mathbf{y}})-\mathcal{S}_{2}^{\kappa}(\boldsymbol{q} ; \widehat{\mathbf{x}}, \widehat{\mathbf{y}})}{\left|\boldsymbol{q}_{\widehat{\mathbf{x}}}-\boldsymbol{q}_{\widehat{\mathbf{y}}}\right|^{2}} \mathcal{V}_{l^{\prime}}^{l}(\boldsymbol{\xi} ; \boldsymbol{q}, \widehat{\mathbf{x}}, \widehat{\mathbf{y}}), \\
& \mathcal{W}_{l^{\prime}}^{l}(\boldsymbol{\xi} ; \boldsymbol{q}, \widehat{\mathbf{x}}, \widehat{\mathbf{y}})=\frac{\left(\boldsymbol{q}_{\widehat{\mathbf{x}}}-\boldsymbol{q}_{\hat{\mathbf{y}}}\right) \cdot\left(\boldsymbol{\xi}_{\widehat{\mathbf{x}}}-\boldsymbol{\xi}_{\widehat{\mathbf{y}}}\right)}{\left|\boldsymbol{q}_{\widehat{\mathbf{x}}}-\boldsymbol{q}_{\hat{\mathbf{y}}}\right|^{2}}\left(\boldsymbol{q}_{\widehat{\mathbf{x}}}-\boldsymbol{q}_{\widehat{\mathbf{y}}}\right) \cdot\left(\boldsymbol{t}_{l^{\prime}}(\boldsymbol{q} ; \widehat{\mathbf{y}}) \times \boldsymbol{t}_{3-l}(\boldsymbol{q} ; \widehat{\mathbf{x}})\right)
\end{aligned}
$$

with

$$
\begin{aligned}
\mathcal{V}_{l^{\prime}}^{l}(\boldsymbol{\xi} ; \boldsymbol{q}, \widehat{\mathbf{x}}, \widehat{\mathbf{y}})= & \left(\boldsymbol{\xi}_{\widehat{\mathbf{x}}}-\boldsymbol{\xi}_{\widehat{\mathbf{y}}}\right) \cdot\left(\boldsymbol{t}_{l^{\prime}}(\boldsymbol{q} ; \widehat{\mathbf{y}}) \times \boldsymbol{t}_{3-l}(\boldsymbol{q} ; \widehat{\mathbf{x}})\right)+\left(\boldsymbol{q}_{\widehat{\mathbf{x}}}-\boldsymbol{q}_{\widehat{\mathbf{y}}}\right) \\
& \cdot\left(\boldsymbol{t}_{\iota^{\prime}}(\boldsymbol{\xi} ; \widehat{\mathbf{y}}) \times \boldsymbol{t}_{3-l}(\boldsymbol{q} ; \widehat{\mathbf{x}})+\boldsymbol{t}_{\iota^{\prime}}(\boldsymbol{q} ; \widehat{\mathbf{y}}) \times \boldsymbol{t}_{3-l}(\boldsymbol{\xi} ; \widehat{\mathbf{x}})\right)
\end{aligned}
$$

The operator $\partial_{\boldsymbol{q}} \widehat{C}_{\kappa}\left[\boldsymbol{q}, \boldsymbol{j}_{\boldsymbol{s}}\right]$ can then be rewritten as

$$
\begin{aligned}
& \left(\partial_{\boldsymbol{q}} \widehat{C}_{\kappa}\left[\boldsymbol{q}, \boldsymbol{j}_{\boldsymbol{s}}\right] \boldsymbol{\xi}\right)(\widehat{\mathbf{x}})=\kappa \sum_{l=1}^{2}(-1)^{l} \boldsymbol{e}^{l}(\widehat{\mathbf{x}}) \sum_{l^{\prime}=1}^{2} \int_{\mathbb{S}^{2}} \frac{R(\boldsymbol{q} ; \widehat{\mathbf{x}}, \widehat{\mathbf{y}})}{|\widehat{\mathbf{x}}-\widehat{\mathbf{y}}|}{ }^{\mathrm{s}} \mathcal{C}_{\kappa, l^{\prime}}^{l}(\boldsymbol{\xi} ; \boldsymbol{q}, \widehat{\mathbf{x}}, \widehat{\mathbf{y}}) \boldsymbol{j}_{\boldsymbol{s}}^{\boldsymbol{i}^{\prime}}(\widehat{\mathbf{y}}) d s(\widehat{\mathbf{y}}) \\
& +i \kappa \sum_{l=1}^{2}(-1)^{l} \boldsymbol{e}^{l}(\widehat{\mathbf{x}}) \sum_{l^{\prime}=1}^{2} \int_{\mathbb{S}^{2}}{ }^{r} \mathcal{C}_{\kappa, l^{\prime}}^{l}(\boldsymbol{\xi} ; \boldsymbol{q}, \widehat{\mathbf{x}}, \widehat{\mathbf{y}}) \boldsymbol{j}_{\boldsymbol{s}}^{\boldsymbol{l}^{\prime}}(\widehat{\mathbf{y}}) d s(\widehat{\mathbf{y}}) \\
& -\frac{1}{\kappa} \operatorname{curl}_{\mathbb{S}^{2}} \int_{\mathbb{S}^{2}} \frac{R(\boldsymbol{q} ; \widehat{\mathbf{x}}, \widehat{\mathbf{y}})}{|\widehat{\mathbf{x}}-\widehat{\mathbf{y}}|} \mathrm{s}^{\mathrm{s}} \mathcal{S}_{\kappa}(\boldsymbol{\xi} ; \boldsymbol{q}, \widehat{\mathbf{x}}, \widehat{\mathbf{y}}) \operatorname{div}_{\mathbb{S}^{2} \boldsymbol{j}_{\boldsymbol{s}}}(\widehat{\mathbf{y}}) d s(\widehat{\mathbf{y}}) \\
& -\frac{i}{\kappa} \operatorname{curl}_{\mathbb{S}^{2}} \int_{\mathbb{S}^{2}}{ }^{r} \mathcal{S}_{\kappa}(\boldsymbol{\xi} ; \boldsymbol{q}, \widehat{\mathbf{x}}, \widehat{\mathbf{y}}) \operatorname{div}_{\mathbb{S}^{2} j_{\boldsymbol{s}}}(\widehat{\mathbf{y}}) d s(\widehat{\mathbf{y}}),
\end{aligned}
$$




$$
{ }^{s} \mathcal{S}_{\kappa}(\boldsymbol{\xi} ; \boldsymbol{q}, \widehat{\mathbf{x}}, \widehat{\mathbf{y}})=-\left(\frac{\mathcal{S}_{1}^{\kappa}(\boldsymbol{q} ; \widehat{\mathbf{x}}, \widehat{\mathbf{y}})}{\left|\boldsymbol{q}_{\widehat{\mathbf{x}}}-\boldsymbol{q}_{\widehat{\mathbf{y}}}\right|^{2}}+\kappa \mathcal{S}_{2}^{\kappa}(\boldsymbol{q} ; \widehat{\mathbf{x}}, \widehat{\mathbf{y}})\right)\left(\boldsymbol{q}_{\widehat{\mathbf{x}}}-\boldsymbol{q}_{\widehat{\mathbf{y}}}\right) \cdot\left(\boldsymbol{\xi}_{\widehat{\mathbf{x}}}-\boldsymbol{\xi}_{\widehat{\mathbf{y}}}\right),
$$

with

$$
\begin{aligned}
& { }^{r} \mathcal{S}_{\kappa}(\boldsymbol{\xi} ; \boldsymbol{q}, \widehat{\mathbf{x}}, \widehat{\mathbf{y}})=\frac{\kappa \mathcal{S}_{1}^{\kappa}(\boldsymbol{q} ; \widehat{\mathbf{x}}, \widehat{\mathbf{y}})-\mathcal{S}_{2}^{\kappa}(\boldsymbol{q} ; \widehat{\mathbf{x}}, \widehat{\mathbf{y}})}{\left|\boldsymbol{q}_{\widehat{\mathbf{x}}}-\boldsymbol{q}_{\widehat{\mathbf{y}}}\right|^{2}}\left(\boldsymbol{q}_{\widehat{\mathbf{x}}}-\boldsymbol{q}_{\widehat{\mathbf{y}}}\right) \cdot\left(\boldsymbol{\xi}_{\widehat{\mathbf{x}}}-\boldsymbol{\xi}_{\widehat{\mathbf{y}}}\right) \\
& { }^{\mathrm{s}} \mathcal{C}_{\kappa, l^{\prime}}^{l}(\boldsymbol{\xi} ; \boldsymbol{q}, \widehat{\mathbf{x}}, \widehat{\mathbf{y}})={ }^{\mathrm{s}} \mathcal{S}_{\kappa}(\boldsymbol{\xi} ; \boldsymbol{q}, \widehat{\mathbf{x}}, \widehat{\mathbf{y}}) \boldsymbol{t}_{l^{\prime}}(\boldsymbol{q} ; \widehat{\mathbf{y}}) \times \boldsymbol{t}_{3-l}(\boldsymbol{q} ; \widehat{\mathbf{x}})+\mathcal{S}_{1}^{\kappa}(\boldsymbol{q} ; \widehat{\mathbf{x}}, \widehat{\mathbf{y}}) \widetilde{\mathcal{V}}_{l^{\prime}}^{l}(\boldsymbol{\xi} ; \boldsymbol{q}, \widehat{\mathbf{x}}, \widehat{\mathbf{y}}), \\
& { }^{r} \mathcal{C}_{\kappa, l^{\prime}}^{l}(\boldsymbol{\xi} ; \boldsymbol{q}, \widehat{\mathbf{x}}, \widehat{\mathbf{y}})={ }^{\mathrm{r}} \mathcal{S}_{\kappa}(\boldsymbol{\xi} ; \boldsymbol{q}, \widehat{\mathbf{x}}, \widehat{\mathbf{y}}) \boldsymbol{t}_{l^{\prime}}(\boldsymbol{q} ; \widehat{\mathbf{y}}) \times \boldsymbol{t}_{3-l}(\boldsymbol{q} ; \widehat{\mathbf{x}})+\mathcal{S}_{2}^{\kappa}(\boldsymbol{q} ; \widehat{\mathbf{x}}, \widehat{\mathbf{y}}) \widetilde{\mathcal{V}}_{l^{\prime}}^{\prime}(\boldsymbol{\xi} ; \boldsymbol{q}, \widehat{\mathbf{x}}, \widehat{\mathbf{y}}), \\
& \widetilde{\mathcal{V}}_{l^{\prime}}^{l}(\boldsymbol{\xi} ; \boldsymbol{q}, \widehat{\mathbf{x}}, \widehat{\mathbf{y}})=\boldsymbol{t}_{l^{\prime}}(\boldsymbol{q} ; \widehat{\mathbf{y}}) \times \boldsymbol{t}_{3-l}(\boldsymbol{\xi} ; \widehat{\mathbf{x}})+\boldsymbol{t}_{l^{\prime}}(\boldsymbol{\xi} ; \widehat{\mathbf{y}}) \times \boldsymbol{t}_{3-l}(\boldsymbol{q} ; \widehat{\mathbf{x}}) .
\end{aligned}
$$

Next, we introduce a change of coordinate system, e.g. for $\widehat{\mathbf{x}} \in \mathbb{S}^{2}$ we consider an orthogonal transformation $T_{\widehat{\mathbf{x}}}$, such that $T_{\widehat{\mathbf{x}}} \widehat{\mathbf{x}}=\widehat{\boldsymbol{\eta}}$, where $\widehat{\boldsymbol{\eta}}={ }^{\mathrm{T}}(0,0,1)$. Introducing an induced linear transformation $\mathcal{T}_{\widehat{\mathbf{x}}}: u(\widehat{\mathbf{y}}) \mapsto u\left(T_{\widehat{\mathbf{x}}}^{-1} \widehat{\mathbf{y}}\right)$ and its bivariate analogue we rewrite the boundary integral operators (4.2), (4.3) in the form where the singularities are moved to only one point, $\widehat{\boldsymbol{\eta}}$. Using the spherical coordinates for the unit sphere parameterization, i.e. $\widehat{\mathbf{z}}=\widehat{\mathbf{z}}\left(\theta^{\prime}, \phi^{\prime}\right)$ the singularity at the point $\widehat{\boldsymbol{\eta}}$ is canceled out by the surface element. Moreover, similarly to [15], it can be shown that the corresponding mappings, e.g. $\left(\theta^{\prime}, \phi^{\prime}\right) \mapsto \mathcal{T}_{\widehat{\mathbf{x}}} R\left(\boldsymbol{q} ; \hat{\boldsymbol{\eta}}, \widehat{\mathbf{z}}\left(\theta^{\prime}, \phi^{\prime}\right)\right) \mathcal{T}_{\widehat{\mathbf{x}}} \mathrm{s} \mathcal{M}_{\kappa, l^{\prime}}^{l}(\boldsymbol{\xi} ; \boldsymbol{q}, \widehat{\boldsymbol{\eta}}, \widehat{\mathbf{z}})$, are smooth.

The numerical integration formula over the unit sphere of continuous function is performed via the Gauss trapezoidal product rule, [40]. For the integrals with weakly singular kernels, we apply the numerical quadrature which is based on the fact that the scalar spherical harmonics are eigenfunctions of the single layer potential on the sphere, [8]. The hypersingular part of the electromagnetic double layer boundary integral operator is computed by integration by parts and employing surface derivatives of the vector spherical harmonics, [33].

The Gauss trapezoidal product rule is exact for the scalar spherical harmonics of order less than or equal to $2 n+1$. It induces the discrete inner product on the space $\mathbb{P}_{n}$ of all scalar spherical harmonics of degree less than or equal to $n$ using which we can define the projection on $\mathbb{P}_{n}$. The inner product of two of tangential vector spherical harmonics of degree less than or equal to $n$ belongs to $\mathbb{P}_{2 n+1}$. This induces the discrete inner product on the space $\mathbb{T}_{n}$ of finite dimension $2(n+1)^{2}-2$ generated by the orthonormal tangential vector spherical harmonics of degree less than or equal to $n$. Hence, we can define the corresponding projection on $\mathbb{T}_{n} \subset \boldsymbol{H}_{\mathrm{div}}^{-\frac{1}{2}}\left(\mathbb{S}^{2}\right)$. Having splitted off the singularities in the kernels, we approximate all operators by replacing the continuous part of the corresponding integrand by its projection onto the space $\mathbb{P}_{n^{\prime}}, n^{\prime}=2 n+1$, the density function is sought in the $\mathbb{T}_{n}$ space and the shape function is sought in the space or real-valued scalar spherical harmonics, $\mathbb{H}_{N_{\text {star }}} \subset \mathcal{Q}_{\text {star }} \subset H^{s}\left(\mathbb{S}^{2}, \mathbb{R}\right)$, where $N_{\text {star }} \leqslant n$. The semi-discrete version of the first equation in (2.9) is projected onto the space $\mathbb{T}_{n}$ and by taking the scalar product with tangential vector spherical harmonics the resulting integral equation is discretized into $2 \times\left((n+1)^{2}-1\right)$ complex-valued algebraic equations for $2 \times\left((n+1)^{2}-1\right)$ complex-valued and $\left(N_{\text {star }}+1\right)^{2}$ real-valued unknown coefficients. The semi-discrete version of the second equation in (2.9) is evaluated at $2 \times\left(N_{m s r}+1\right)^{2}$ measurement points. Finally, we obtain a fully discrete system of $4\left[(n+1)^{2}-1+\left(N_{m s r}+1\right)^{2}\right]$ real-valued equations for $4\left((n+1)^{2}-1\right)+\left(N_{\text {star }}+1\right)^{2}$ real-valued unknown coefficients. The procedure extends in an obvious way to the case of $m>1$ incident plane waves. 


\section{Numerical experiments}

The synthethic data were obtained by solving the boundary integral equation based on Stratton-Chu representation formula with $n_{s y n}=20$. In the numerical algorithm we choose the regularizing operator $\widehat{\Lambda}$ as following [33],

$$
\widehat{\Lambda}=\operatorname{curl}_{\mathbb{S}^{2}}\left(-\Delta_{\mathbb{S}^{2}}\right)^{-1} \operatorname{div}_{\mathbb{S}^{2}}+\operatorname{grad}_{\mathbb{S}^{2}}\left(-\Delta_{\mathbb{S}^{2}}\right)^{-2} \operatorname{curl} \mathbb{S}^{2}
$$

and the parameter $\eta=1$ in (2.1). We present the reconstruction results for one concave obstacle and one convex obstacle: bean(2.0) and rounded hexahedron $(p=10)$, with parametric representation given in [15] and [38], correspondingly. The diameter of the obstacles is roughly 2. Since we consider scattering for frequencies in the resonance region, we choose the wavenumber $\kappa$ such that the wavelength $\lambda=2 \pi / \kappa$ is of a comparable size to the diameter of the obstacle $\Omega$, e.g. we study two cases $\kappa=\pi$ and $\kappa=\pi / 2$. The obstacle is illuminated by only one incident plane wave with the polarization $p={ }^{\top}(1,0,0)$ and the direction $d={ }^{\mathrm{T}}(0,0,1)$. As an initial guess for all reconstruction we consider a unit ball centered at the origin. The parameters for the problem discretization were chosen as follows: $n=15, N_{\text {star }}=15, N_{m s r}=7$. The regularization parameters in (2.11) are selected as $\beta_{N}=0.01\left(\frac{2}{3}\right)^{N}, \alpha_{N}=0.5\left(\frac{2}{3}\right)^{N}$ and $s=2.1$-index of the Sobolev space used for the parametrization of the unknown shape. The iterations are terminated according to the Morozov's discrepency principle (2.12) with $\tau=1.1$. Figure 1 exhibits behaviours of the residual at each iteration step for two different object bean('b') and hexahedron(' $h$ '), two wave numbers and noise levels of $1 \%$ and $5 \%$. From the graph it can be concluded that the best reconstructions are obtained for the noise level of $1 \%$ and the case when the diameter of the obstacle is equal to the wavelength, i.e. $\kappa=\pi$. The worst reconstructions are gained when we increase the noise level and change the wavelenth at the same time. In figure 2 we show the corresponding results. As indicated in [15], calculation of scattered field for the ellipsoidal obstacles require fewer degrees of freedom compared to the bean, therefore it is natural that the reconstruction of the convex rounded rectangle is more accurate than the reconstruction of the concave object. The reconstruction improves and the number of iterations decreases with increase in the number of incident plane waves. In figure 3 the reconstructions of the obstacle illuminated by 6 incident plane waves from top, bottom, front, back, left and right are shown.

\section{Conclusion and perspectives}

In this paper we established the material derivative analysis of the boundary integral operators arising in the potential theory of time-harmonic electromagnetic waves. Among all the proposed approaches [8, 11, 39, 43], the use of the Piola transform of the boundary parametrisations greatly simplifies both the theoretical and numerical investigations. These new results allowed us to propose a new inverse scattering algorithm, based on previous investigations in acoustics [26, 27], for solving shape reconstruction problems in electromagnetism.

A promising advantage of the inverse scattering algorithm presented in this paper is to avoid numerous numerical solutions at each iteration step of exterior boundary value problems that are very time consuming. The method presented in section 2 gives an alternative approach to the adjoint state method usually used to compute the Fréchet derivatives for solving any inverse obstacle scattering problems via geometric optimization methods. This new algorithm coupled with topological optimization tools will allow us to investigate and 


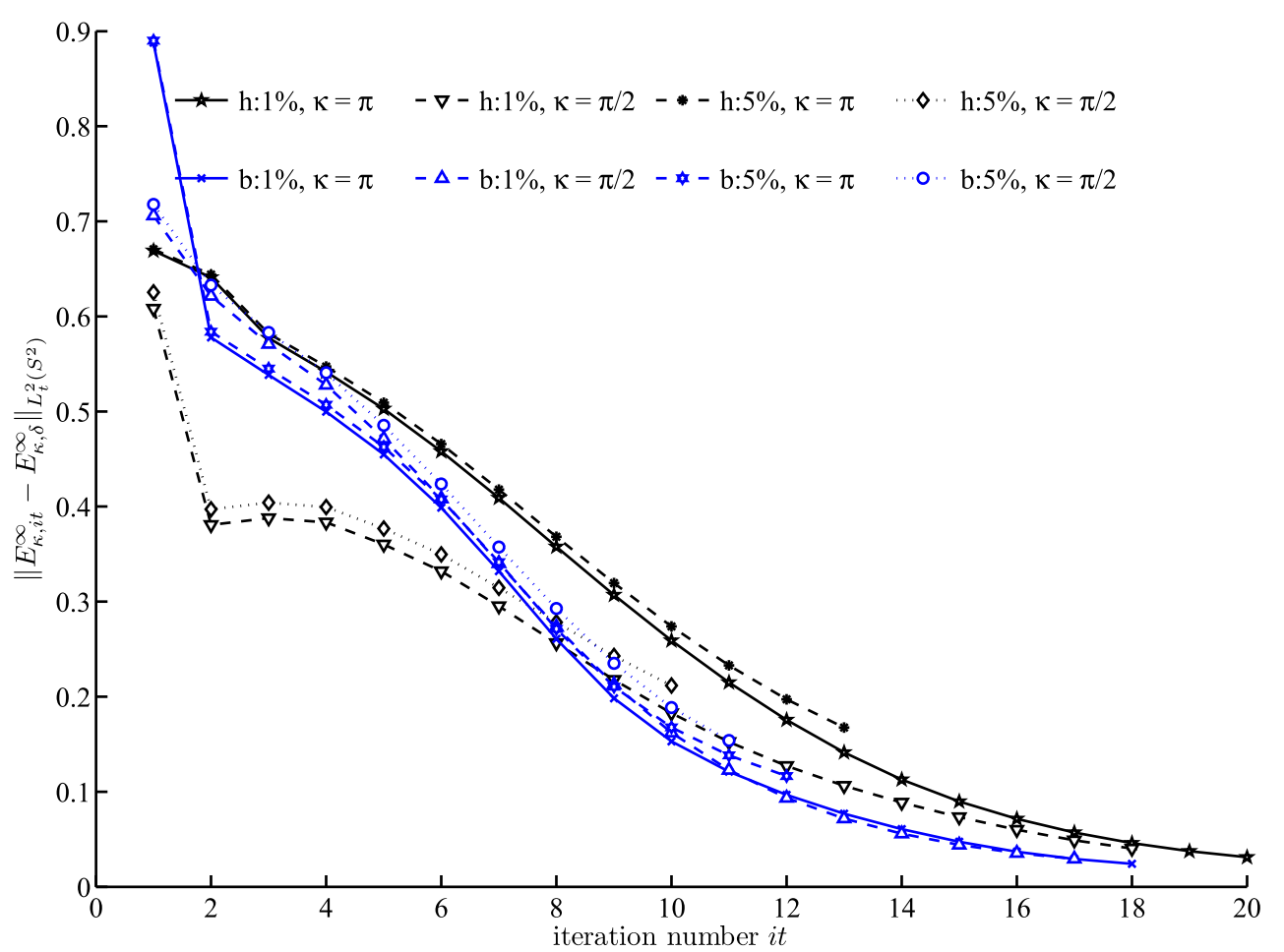

Figure 1. Numerical convergence.
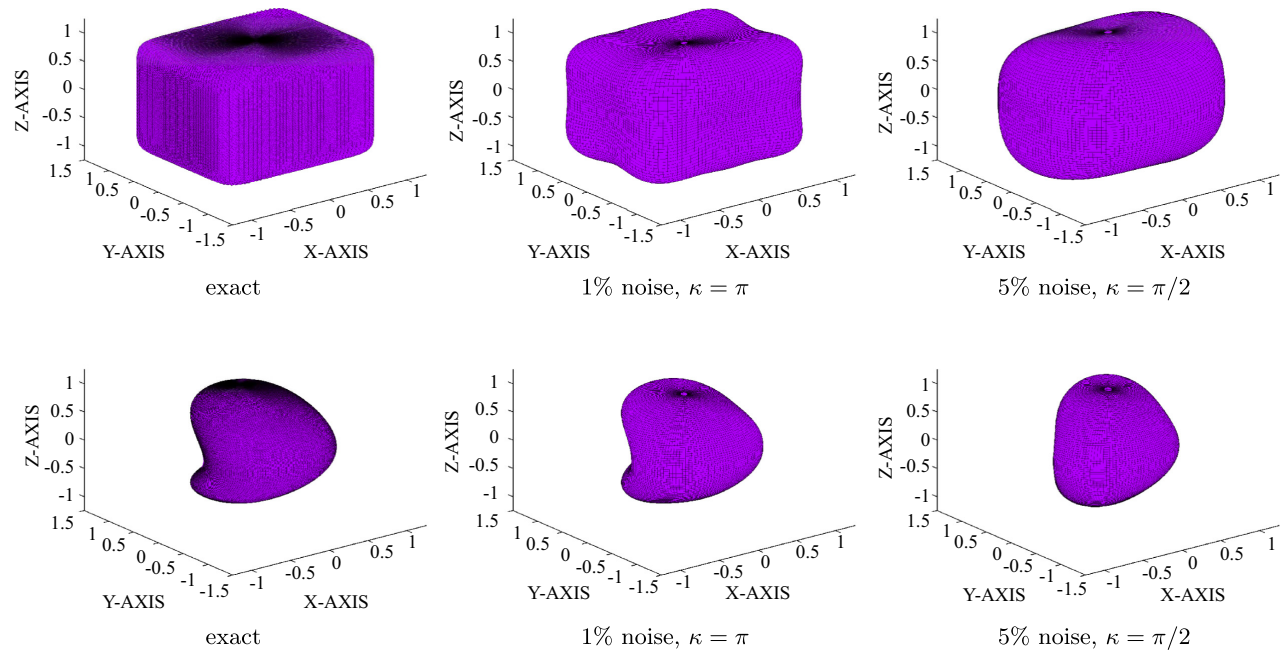

Figure 2. Reconstructions of the obstacles for one incident plane wave.

develop in forthcoming papers new fast computational methods for solving more challenging inverse obstacle scattering problems. That are the location and the shape reconstruction of multiple scatterers with various kind of boundary conditions [6, 7]. 


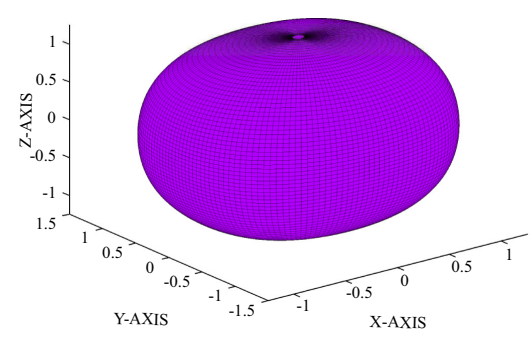

2 iterations

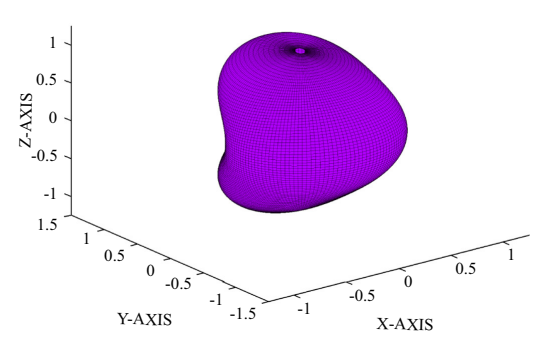

5 iterations

Figure 3. Reconstructions for 6 incident plane waves, $5 \%$ noise, $\kappa=\pi / 2$.

\section{Acknowledgments}

The authors gratefully acknowledges Professor Dr Thorsten Hohage from the Institute of Numerical and Applied Mathematics at the University of Goettingen for providing the inversion toolbox he developed with Matlab programing language [21, 22].

\section{References}

[1] Adams R A 1975 Sobolev spaces Pure and Applied Mathematics vol 65 (New York: Academic)

[2] Atkinson K E 1982 The numerical solution of Laplace's equation in three dimensions: I. SIAM J. Numer. Anal. 19 263-74

[3] Bakushinskiǔ A B 1992 On a convergence problem of the iterative-regularized Gauss-Newton method Comput. Math. Math. Phys. 32 1503-9

[4] Buffa A and Ciarlet P Jr. 2001 On traces for functional spaces related to Maxwell's equations: II. Hodge decompositions on the boundary of Lipschitz polyhedra and applications Math. Methods Appl. Sci. 24 31-48

[5] Buffa A, Hiptmair R, von Petersdorff T and Schwab C 2003 Boundary element methods for Maxwell transmission problems in Lipschitz domains Numer. Math. 95 459-85

[6] Cakoni F and Kress R 2013 Integral equation methods for the inverse obstacle problem with generalized impedance boundary condition Inverse Problems 2901500519

[7] Carpio A, Johansson B T and Rapún M-L 2010 Determining planar multiple sound-soft obstacles from scattered acoustic fields J. Math. Imaging Vision 36 185-99

[8] Colton D and Kress R 2013 Inverse acoustic and electromagnetic scattering theory Appl. Math. Sci. vol 93 3rd edn (New York: Springer)

[9] Costabel M and Le Louër F 2011 On the Kleinman-Martin integral equation method for electromagnetic scattering by a dielectric body SIAM J. Appl. Math. 71 635-56

[10] Costabel M and Le Louër F 2012 Shape derivatives of boundary integral operators in electromagnetic scattering: I. Shape differentiability of pseudo-homogeneous boundary integral operators Integral equations and Operator Theory 72 509-35

[11] Costabel M and Le Louër F 2012 Shape derivatives of boundary integral operators in electromagnetic scattering: II. Application to scattering by a homogeneous dielectric obstacle Integral Equations and Operator Theory 72 509-35

[12] de La Bourdonnaye A 1993 Décomposition de $H-1 / 2 \operatorname{div}(\Gamma)$ et nature de l'opérateur de Steklov-Poincaré du problème extérieur de l'électromagnétisme C. R. Acad. Sci. Paris Sér. I Math. 316 369-72

[13] El Bouajaji M, Antoine X and Geuzaine C 2014 Approximate local magnetic-to-electric surface operators for time-harmonic Maxwell's equations J. Comput. Phys. 279 241-60

[14] Gao Z M, Ma Y C and Zhuang H W 2008 Shape optimization for Navier-Stokes flow Inverse Probl. Sci. Eng. 16 583-616

[15] Ganesh M and Graham I G 2004 A high-order algorithm for obstacle scattering in three dimensions J. Comput. Phys. 198 211-42 
[16] Ganesh M and Hawkins S C 2007 A hybrid high-order algorithm for radar cross section computations SIAM J. Sci. 29 1217-43

[17] Ganesh M and Hawkins S C 2008 A high-order tangential basis algorithm for electromagnetic scattering by curved surfaces J. Comput. Phys. 227 4543-62

[18] Graham I G and Sloan I H 2002 Fully discrete spectral boundary integral methods for Helmholtz problems on smooth closed surfaces in $\mathbb{R}^{3}$ Numer. Math. 92 289-323

[19] Haddar H and Kress R 2004 On the Fréchet derivative for obstacle scattering with an impedance boundary condition SIAM J. Appl. Math. 65 194-208

[20] Harbrecht $\mathrm{H}$ and Hohage T 2007 Fast methods for three-dimensional inverse obstacle scattering problems J. Integral equations Appl. 19 237-60

[21] Hohage T 1997 Logarithmic convergence rates of the iteratively regularized Gauss-Newton method for an inverse potential and an inverse scattering problem Inverse Problems 13 1279-99

[22] Hohage T 1999 Iterative Methods in Inverse Obstacle Scattering: Regularization Theory of Linear and Nonlinear Exponentially Ill-Posed Problems PhD Thesis University of Linz

[23] Hohage T and Le Louër F 2013 A Spectrally Accurate Method for the Dielectric Obstacle Scattering Problem and Applications to the Inverse Problem preprint, http://num.math.unigoettingen.de/preprints/files/2013-20.pdf

[24] Hohage T and Schormann C 1998 A Newton-type method for a transmission problem in inverse scattering Inverse Problems 14 1207-27

[25] Ivanyshyn O and Johansson B T 2008 Boundary integral equations for acoustical inverse soundsoft scattering J. Inverse Ill-Posed Probl 16 65-78

[26] Ivanyshyn $\mathrm{O}$ and Johansson T 2007 Nonlinear integral equation methods for the reconstruction of an acoustically sound-soft obstacle J. Integral equations Appl. 19 289-308

[27] Ivanyshyn O and Kress R 2010 Identification of sound-soft 3D obstacles from phaseless data Inverse Probl. Imaging 4 131-49

[28] Ivanyshyn O, Kress R and Serranho P 2010 Huygens' principle and iterative methods in inverse obstacle scattering Adv. Comput. Math. 33 413-29

[29] Kirsch A 1993 The domain derivative and two applications in inverse scattering theory Inverse Problems 9 81-96

[30] Kress R 2001 Electromagnetic waves scattering: scattering by obstacles Scattering ed E R Pike and P C Sabatier (London: Academic) pp 191-210

[31] Kress R 2002 Uniqueness in inverse obstacle scattering for electromagnetic waves Proc. of the URSI General Assembly, Maastricht

[32] Kress R and Rundell W 2005 Nonlinear integral equations and the iterative solution for an inverse boundary value problem Inverse Problems 21 1207-23

[33] Le Louër F 2014 Spectrally accurate numerical solution of hypersingular boundary integral equations for three-dimensional electromagnetic wave scattering problems J. Comput. Phys. 275 662-6

[34] Le Louër F 2015 A domain derivative-based method for solving elastodynamic inverse obstacle scattering problems Inverse Problems 31115006

[35] Levadoux D, Millot F and Pernet S 2010 New trends in the preconditioning of integral equations of electromagmetism Mathematics in Industry 14 383-94

[36] Liu H 2008 A global uniqueness for formally determined inverse electromagnetic obstacle scattering Inverse Problems 2403501813

[37] Jean-Claude N 2001 Acoustic and electromagnetic equations Appl. Math. Sci. vol 144 (New York: Springer) Integral representations for harmonic problems

[38] Onaka S 2006 Simple equations giving shapes of various convex polyhedra: the regular polydedra and polyhedra composed of crystallographically low-index planes Phil. Mag. Lett. 86 175-83

[39] Pieper M 2008 Nonlinear integral equations for an inverse electromagnetic scattering problem J. Phys. 124

[40] Pieper M 2009 Vector hyperinterpolation on the sphere J. Approx. Theory 156 173-86

[41] Plotnikov P I and Sokolowski J 2013 Optimal shape control of airfoil in compressible gas flow governed by Navier-Stokes equations Evol. Equ. Control Theory 2 495-516

[42] Potthast R 1994 Fréchet differentiability of boundary integral operators in inverse acoustic scattering Inverse Problems 10 431-47 
[43] Potthast R 1996 Domain derivatives in electromagnetic scattering Math. Methods Appl. Sci. 19 $1157-75$

[44] Steinbach O and Windisch M 2009 Modified combined field integral equations for electromagnetic scattering SIAM J. Numer. Anal. 47 1149-67

[45] Wienert L 1990 Die numerische Approximation von Randintegraloperatoren für die Helmholtzgleichung im $\mathbb{R} 3$ Dissertation, Univ. Göttingen, 92 pages. 